\title{
A Tutorial on Modeling and Analysis of Dynamic Social Networks. Part II. ${ }^{\hat{w}}$
}

\author{
Anton V. Proskurnikov ${ }^{\mathrm{a}, \mathrm{b}, \mathrm{c}, *}$, Roberto Tempo ${ }^{\mathrm{d}}$ \\ ${ }^{a}$ Delft Center for Systems and Control, Delft University of Technology, Delft, The Netherlands \\ ${ }^{b}$ Institute for Problems of Mechanical Engineering of the Russian Academy of Sciences, St. Petersburg, Russia \\ ${ }^{c}$ ITMO University, St. Petersburg, Russia \\ ${ }^{d}$ CNR-IEIIT, Politecnico di Torino, Torino, Italy
}

\begin{abstract}
Recent years have witnessed a significant trend towards filling the gap between Social Network Analysis (SNA) and control theory. This trend was enabled by the introduction of new mathematical models describing 'dynamics of social groups, the development of algorithms and software for data analysis and the tremendous progress in understanding complex networks and multi-agent systems (MAS) dynamics. The aim of this tutorial is to highlight a novel chapter of control theory, dealing with dynamic models of social networks and processes over them, to the attention of the broad research community. In its first part [1], we have considered the most classical models of social dynamics, which have anticipated and to a great extent inspired the recent extensive studies on MAS and complex networks. This paper is the second part of the tutorial, and it is focused on more recent models of social processes that have been developed concurrently with MAS theory. Future perspectives of control in social and techno-social systems are also discussed.
\end{abstract}

'Keywords: Social network, opinion dynamics, multi-agent systems, distributed algorithms.

\section{Introduction}

Originating from the early studies on sociome'try [2, 3], Social Network Analysis (SNA) has quickly 'grown into an interdisciplinary science [4 7] that has found applications in political sciences [8, 9], medicine [10], economics [11, 12], crime prevention and security [13, 14]. The recent breakthroughs in algorithms and software for big data analysis have made SNA an efficient tool to study online social networks and media [15, 16] with millions of users. The development of SNA has inspired many important concepts of modern network science [17 20] such as cliques and communities, centrality measures, resilience, graph's density and clustering coefficient.

Employing many mathematical and algorithmic tools, SNA has however benefited little from the recent progress in systems and control [21 23]. The realm of social sciences has remained almost un-

\footnotetext{
The paper is supported by Russian Science Foundation (RSF) grant 14-29-00142, hosted by IPME RAS.

${ }^{*}$ Corresponding author

Email address: anton.p.1982@ieee.org (Anton V. Proskurnikov)
}

touched by control theory, despite the long-term studies on social group dynamics [24-26] and "sociocybernetics" 27 30]. This gap between SNA and control can be explained, to a great extent, by the lack of dynamic models of social processes and mathematical armamentarium for their analysis. Focusing on topological properties of networks, SNA and network science have paid much less attention to dynamics over them, except for some special processes such as e.g. random walks, branching and queueing processes, percolation and contagion dynamics [19, 20].

The recent years have witnessed an important tendency towards filling the gap between SNA and control theory, enabled by the rapid progress in multiagent systems and dynamic networks. The emerging branch of control theory, studying social processes, is very young and even has no name yet. However, the interest of sociologists to this new area and understanding that "coordination and control of social systems is the foundational problem of sociology" [31] leaves no doubt that it should become a key instrument to examine social networks and dynamics over them. Without aiming to provide a exhaustive survey of "social control theory" at its dawn, this tutorial fo-

April 3, 2018 
cuses on the most "mature" results, primarily dealing with mechanisms of opinion formation [31 36].

In the first part of this tutorial [1], the most classical models of opinion formation have been discussed that have anticipated and inspired the "boom" in multi-agent and networked control, witnessed by the past decades. This paper is the second part of the tutorial and deals with more recent dynamic models, taking into account effects of time-varying graphs, homophily, negative influence, asynchronous interactions and quantization. The theory of such models and multi-agent control have been developed concurrently, inspiring and reinforcing each other.

Whereas analysis of the classical models addressed in [1] is mainly based on linear algebra and matrix analysis, the models discussed in this part of the tutorial require more sophisticated and diverse mathematical tools. The page limit makes it impossible to include the detailed proofs of all results discussed in this part of the tutorial; for many of them, we have to omit the proofs or provide only their brief sketches.

The paper is organized as follows. Section 2 introduces preliminary concepts and some notation used throughout the paper. Section 3 considers basic results, concerned with properties of the non-stationary French-DeGroot and Abelson models. In Section 4 we consider bounded confidence models, where the interaction graph is opinion-dependent. Section 5 is devoted to dynamic models based on asynchronous gossiping interactions. Section 6 introduces some models, exploiting the idea of negative influence. Section 7 concludes the tutorial.

\section{Preliminaries and notation}

In this section we introduce some notation; basic concepts regarding opinion formation modeling are also recollected for the reader's convenience.

\subsection{Notation}

We use $m: n$ to denote the set $\{m, m+1, \ldots, n\}$ (here $m, n$ are integer and $m \leq n$ ). Given a vector $x \in \mathbb{R}^{n},|x|$ stands for its Euclidean norm $|x|=\sqrt{x^{\top} x}$.

Each non-negative matrix $A=\left(a_{i j}\right)_{i, j \in V}$ corresponds to the weighted graph $\mathcal{G}[A]=(V, E[A], A)$, whose arcs represent positive entries of $A: a_{i j}>0$ if and only if $(j, i) \in E(A)$. Being untypical for graph theory (where the entry $a_{i j}>0$ is encoded by the arc $(i, j)$ ), this notation is convenient in social dynamics modeling [1] and multi-agent control [37, 38].
Dealing with algorithms' complexity, we use standard Landau-Knuth notation [39]. Given two positive functions $f(n), g(n)$ of the natural argument $n$, $g(n)=O(f(n))$ stands for the estimate $|g(n)| \leq$ $C|f(n)|$, where $C$ is some constant, and $f(n)=$ $\Omega(g(n))$ means that $\varliminf_{n \rightarrow \infty} f(n) / g(n)>0$ (i.e. $f\left(n_{k}\right) \geq$ $c_{0} g\left(n_{k}\right)$ for a constant $c_{0}>0$ and a sequence $n_{k} \rightarrow \infty$.

\subsection{Agent-based modeling of opinion evolution}

From the sociological viewpoint [31], an individual's opinion stands for his/her cognitive orientation towards some object (e.g. issue, event, action or another individual). Mathematically, opinions are scalar or vector quantities of interest, associated with social actors. Depending on the specific model, opinions may represent signed attitudes $40-$ 42], subjective certainties of belief [43, 44] or probabilities [45, 46]. In this tutorial, we deal with models where opinions can attain a continuum of values and are represented by real numbers or vectors. Dynamics of real-valued opinions obey ordinary differential or recurrent equation and are much better studied by the systems and control community than the evolution of discrete (finite-valued) opinions. For this reason, many important models with finite-valued opinions [47 55] are beyond the scope of this tutorial.

Historically, the first approach to social dynamics modeling originates from mathematical biology 5659], portraying social behaviors as interactions of multiple "species" or compartments [60]. Dealing with a social group, a compartment is a subgroup whose members are featured by some behavior or hold the same position on some issue. Interacting as indecomposable entities, compartments can grow or decline. The models describing these processes are called compartmental and broadly used in mathematical biology and evolutionary game theory [60 62], as exemplified by the SIR/SIS models of epidemic spread and the Lotka-Volterra predator-prey dynamics. Compartmental models describe how the distribution of individuals between the compartments evolves, paying no attention to behaviors of specific social actors. This statistical approach is typical for sociodynamics 63 65], representing the state of a society by a point in some configuration space and has lead to statistical model of opinion formation, describing how the distribution of opinions evolves over time. Similar in spirit to models arising in continuum mechanics, such models are often referred to as Eulerian [66 68] or continuum [69 71] opinion dynamics. 
In this tutorial, we focus on agent-based models of opinion formation, describing how the opinion of each individual social actor, or agent, evolves under the influence of the remaining individuals. The collective behavior of a social group is constituted by the numerous individual behaviors. Such "bottomup" models of social dynamics, called also aggregative [72], are similar in spirit to agent-based models of self-organization in complex physical and biological systems [73 75]. Unlike statistical models, adequate for very large social groups, agent-based models can describe both small-size and large-scale communities. Throughout this paper, we deal with a closed community of $n \geq 2$ agents, indexed 1 through $n$.

\subsection{Models of consensus and Abelson's puzzle}

As have been discussed in the first part of this tutorial [1], the first agent-based model of opinion formation was introduced by French 76] and later studied and extended by Harari [77, 78] and DeGroot [45]. The French-DeGroot model describes the discretetime evolution of the agents opinions $x_{1}, \ldots, x_{n} \in \mathbb{R}$, whose stack vector $x(k)=\left(x_{1}(k), \ldots, x_{n}(k)\right)^{\top} \in \mathbb{R}^{n}$ at step $k=0,1, \ldots$ obeys the averaging dynamics

$$
x(k+1)=W x(k), \quad k=0,1, \ldots
$$

where $W=\left(w_{i j}\right)$ is a stochastic matrix. The continuous-time counterpart of (11), proposed by Abelson [40], is the Laplacian flow dynamics [79]

$$
\dot{x}(t)=-L[A] x(t), \quad t \geq 0,
$$

where $A=\left(a_{i j}\right)$ is a non-negative matrix of "contact rates" and $L[A]$ stands for the Laplacian matrix of the corresponding weighted graph [1, 79]. The asymptotic consensus of opinions appears to be the most typical behavior of the systems (11) and (2), the relevant criteria are considered in [1]. At the same time, real social groups often fail to reach consensus and exhibit clustering of opinions and other "irregular" behaviors. This has lead Abelson to the fundamental problem, called the community cleavage problem [31] or Abelson's diversity puzzle [80]: to find mathematical models, able to explain these disagreement effects. The original formulation of Abelson [40] was as follows: "we are naturally lead to inquire what on earth one must assume in order to generate the bimodal outcome of community cleavage studies".

One reason for community cleavage is the absence of connectivity: consensus of opinions in the models (11) and (2) cannot be established when the corresponding interaction graph $\mathcal{G}[W]$ or $\mathcal{G}[A]$ has no directed spanning tree. Although social networks are usually densely connected [81], they may contain some "radical" groups [82], closed to social influence. For instance, consensus is not possible in presence for several stubborn individuals (or zealots) [53, 83], whose opinion remains unchanged $x_{i} \equiv x_{i}(0)$. Further development of this idea naturally leads [1] to the Friedkin-Johnsen theory of social influence networks [84, 85] with "partially stubborn" agents.

Stubborness is however not the only factor leading to the community cleavage; in this part of the tutorial we consider other models of opinion formation where opinions can both converge to consensus or split into several clusters. Many of these models are based on the ideas, proposed in the seminal Abelson's works [40, 72] and extend the classical models (1),(2).

\section{The models by French-DeGroot and Abel- son with time-varying interaction graphs}

Non-stationary counterparts of the models (11) and (2) have been thoroughly studied in regard to consensus and synchronization in multi-agent networks. In this tutorial, only some results are considered that directly related to social dynamics; detailed overview of consensus algorithms can be found e.g. in the recent monographs and surveys [37, 38, 86 90].

\subsection{The time-varying French-DeGroot model}

We start with a time-varying counterpart of (11), where $W$ is replaced by a sequence $(W(k))_{k \geq 0}$

$$
x(k+1)=W(k) x(k), \quad k=0,1, \ldots
$$

Obviously, all solutions to (3) are bounded and the sequences $\min _{i} x_{i}(k)$ and $\max _{i} x_{i}(k), k=0,1, \ldots$, are respectively non-decreasing and non-increasing.

As discussed in [1], even for the static case $W(k) \equiv$ $W$ the opinions do not always converge. For instance, when the graph $\mathcal{G}[W]$ is periodic, the system (11) has a periodic solution. The convergence problem for timevarying system (3) still remains a challenge, and up to now only sufficient convergence conditions have been obtained. One of them is given by the following important result, proved independently in 91 93].

Lemma 1. Let $\delta>0$ exist such that the sequence of $n \times n$ stochastic matrices $(W(k))_{k \geq 0}$ satisfies at any time $k \geq 0$ the following three conditions:

(a) (non-vanishing couplings) $w_{i j}(k) \in\{0\} \cup[\delta, 1]$; 
(b) (self-confidence) $w_{i i}(k) \geq \delta \quad \forall i \in 1: n$;

(c) (type-symmetry) $w_{i j}(k)>0 \Longleftrightarrow w_{j i}(k)>0$.

Then the limit $\bar{x}=\lim _{k \rightarrow \infty} x(k)$ exists for any $x(0) \in$ $\mathbb{R}^{n}$, being an equilibrium point: $W(k) \bar{x}=\bar{x}$ for sufficiently large $k$. If agents $i$ and $j$ interact persistently

$$
\sum_{k=0}^{\infty} w_{i j}(k)=\infty
$$

then their limit opinions coincide $\bar{x}_{i}=\bar{x}_{j}$.

Introducing the undirected graph of persistent interactions $\mathcal{G}_{\infty}=\left(V, E_{\infty}\right)$, whose nodes stand for the agents and $\operatorname{arcs}(i, j)$ connect pairs of persistently interacting agents, the last statement of Lemma 1 can be formulated as follows: in each connected component of $\mathcal{G}_{\infty}$, the opinions reach consensus.

We give a sketch of the proof of Lemma 1, following the ideas from 91] and proposed in [94] for more general systems of recurrent inequalities. It suffices to consider the case of connected graph $\mathcal{G}_{\infty}$. Indeed, if $(i, j) \notin E_{\infty}$, then $w_{i j}(k)>0$ only for finite number of instants $k$ thanks to condition (a). In other words, $k_{0} \geq 0$ exists such that $w_{i j}(k)=0$ for $k>k_{0}$ unless $i$ and $j$ persistently interact. Renumbering the agents, for $k>k_{0}$ the matrix $W(k)$ is block diagonal

$$
W(k)=\left(\begin{array}{ccc}
W_{11}(k) & \ldots & 0 \\
\vdots & \ddots & \vdots \\
0 & \ldots & W_{r r}(k)
\end{array}\right),
$$

where the stochastic submatrices $W_{i i}(k)$ correspond to connected components of $\mathcal{G}_{\infty}$. Hence (3) for $k>k_{0}$ is decoupled into $r$ independent systems.

Let $\mathcal{G}_{\infty}$ be connected and $j_{1}(k), \ldots, j_{n}(k)$ be the permutation of indices, sorting the opinions $x_{1}(k), \ldots, x_{n}(k)$ in the ascending order, that is, $y_{i}(k)=x_{j_{i}(k)}(k)$ satisfy the following inequalities

$\min _{i} x_{i}(k)=y_{1}(k) \leq y_{2}(k) \leq \ldots \leq y_{n}(k)=\max _{i} x_{i}(k)$.

We are going to prove the following statement: for any $k \geq 0$ and $i=1, \ldots, n-1$, there exists $k^{\prime}>k$ (depending on both $k, i)$, satisfying the inequality

$$
y_{i+1}\left(k^{\prime}\right) \leq \delta y_{i}(k)+(1-\delta) y_{n}(k),
$$

where $\delta>0$ is the constant from condition (a). To prove this, divide the agents into two sets $I=$ $\left\{j_{1}(k), \ldots, j_{i}(k)\right\}$ and $J=\left\{j_{i+1}(k), \ldots, j_{n}(k)\right\}$. Since $\mathcal{G}_{\infty}$ is connected, an arc between $I$ and $J$ should exist, and hence there exist $K>k$, such that $w_{q p}(K) \geq \delta$ for some $p \in I, q \in J$. Let $k_{0}$ stand for the minimal such $K$. Since $x_{s}(k) \leq y_{i}(k)$ for any $s \in I$ and the agents from $I$ and $J$ do not interact at times $k, k+1, \ldots, k_{0}-1$, it can be shown that $x_{r}\left(k_{0}\right) \leq$ $y_{i}(k) \forall r \in I$. Also $x_{r}\left(k_{0}\right) \leq y_{n}\left(k_{0}\right) \leq y_{n}(k) \forall r \in J$ since $y_{n}(k)$ is non-increasing in $k$. For $r \in I$, one has

$$
\begin{array}{r}
x_{r}\left(k_{0}+1\right) \leq x_{r}\left(k_{0}\right)+\overbrace{\sum_{l \neq r} w_{r l}\left(k_{0}\right)}^{1-w_{r r}\left(k_{0}\right) \leq 1-\delta}[\underbrace{x_{l}\left(k_{0}\right)}_{\leq y_{n}\left(k_{0}\right)}-x_{r}\left(k_{0}\right)] \leq \\
\quad \leq x_{r}\left(k_{0}\right)+(1-\delta)\left[y_{n}\left(k_{0}\right)-x_{r}\left(k_{0}\right)\right] \leq \\
\leq \delta x_{r}\left(k_{0}\right)+(1-\delta) y_{n}\left(k_{0}\right) \leq \delta y_{i}(k)+(1-\delta) y_{n}(k) .
\end{array}
$$

Recalling that $w_{q p}\left(k_{0}\right) \geq \delta$ and $p \in I$, similarly to the previous inequality one obtains

$$
\begin{array}{r}
x_{q}\left(k_{0}+1\right) \leq w_{q p}\left(k_{0}\right) x_{p}\left(k_{0}\right)+\left(1-w_{q p}\left(k_{0}\right)\right) y_{n}\left(k_{0}\right)= \\
=y_{n}\left(k_{0}\right)-w_{q p}\left(k_{0}\right)\left[y_{n}\left(k_{0}\right)-x_{p}\left(k_{0}\right)\right] \leq \\
\leq \delta x_{p}\left(k_{0}\right)+(1-\delta) y_{n}\left(k_{0}\right) \leq \delta y_{i}(k)+(1-\delta) y_{n}(k) .
\end{array}
$$

Denoting $k^{\prime}=k_{0}+1$, for any index $\rho \in I^{\prime}=I \cup\{q\}$ one has $x_{\rho}\left(k^{\prime}\right) \leq \delta y_{i}(k)+(1-\delta) y_{n}(k)$. Since $I^{\prime}$ contains $i+1$ different indices, one arrives at (4). Since $y_{n}(k)$ is bounded from below and non-increasing, it converges to a limit $y_{n}(k) \rightarrow M_{*}$ as $k \rightarrow \infty$. Passing to the limit as $k \rightarrow \infty$ in (4), the corresponding sequence $k^{\prime}=k^{\prime}(i, k)$ also tends to $\infty$ and thus

$$
\varliminf_{k \rightarrow \infty} y_{i+1}(k) \leq \delta \varliminf_{k \rightarrow \infty} y_{i+1}(k)+(1-\delta) M_{*} .
$$

Applying this to $i=n-1$, one has $M_{*} \leq \underline{\lim }_{k \rightarrow \infty} y_{n-1}(k) \leq \overline{\lim }_{k \rightarrow \infty} y_{n-1}(k) \leq$ $\varlimsup_{k \rightarrow \infty} y_{n}(k)=M_{*}$, and therefore $y_{n-1}(k) \underset{k \rightarrow \infty}{\longrightarrow}$ $M_{*}$. Iterating this procedure for $i=n-2, \ldots, 1$, one proves that $y_{i}(k) \rightarrow M_{*}$, i.e. consensus of opinions is established. Obviously, any consensus vector $c \mathbb{1}_{n}$ is an equilibrium point, which finishes the proof.

The convergence of opinions in (3) can be reformulated in terms of matrix products convergence [93].

Corollary 2. Under the assumptions of Lemma 1, the limit of the matrix products exist

$$
\bar{W}=\lim _{k \rightarrow \infty} W(k) \ldots W(1) W(0) .
$$

Renumbering of the agents, $\bar{W}$ is block-diagonal

$$
\bar{W}=\left(\begin{array}{ccc}
\bar{W}_{11} & \ldots & 0 \\
\vdots & \ddots & \vdots \\
0 & \ldots & \bar{W}_{r r}
\end{array}\right)
$$


where $r$ is the number of connected components in $\mathcal{G}_{\infty}$ and $\bar{W}_{i i}$ are "consensus matrices" $\mathbb{1}_{n_{i}} p_{i}^{\top}$, where $p_{i} \in \mathbb{R}^{n_{i}}$ is a non-negative vector with $p_{i}^{\top} \mathbb{1}_{n_{i}}=1$.

It should be noticed that the consensus criterion from Lemma 1 can be substantially extended [95, 96]. In particular, are reported in the preprint [96], in the case of connected undirected graph $\mathcal{G}_{\infty}$ the typesymmetry condition (c) can be relaxed to its "noninstantaneous" version and (a) can be discarded (assuming (b) to be valid). Many models of opinion dynamics (e.g. time-varying extensions of the FJ model [97]), however, exhibit disagreement instead of consensus, being uncovered by these strong results.

A natural question arises how to measure the rate of convergence in (3) (being, in general, nonexponential). Possible measures for such a rate are the total and the kinetic s-energies [98], defined as

$$
\begin{aligned}
E(s) & =\sum_{\substack{k=0,1,2, \ldots \\
(i, j): w_{i j}(k) \neq 0}}\left|x_{i}(k)-x_{j}(k)\right|^{s} \\
K(s) & =\sum_{k=0}^{\infty} \sum_{i=1}^{n}\left|x_{i}(k+1)-x_{i}(k)\right|^{s} .
\end{aligned}
$$

It is not obvious that $E(s)$ and $K(s)$ are finite for $s \leq 1$, however, under the assumptions of Lemma 1 both series converge $\ell^{2}$ for all $s>0$. Some explicit estimates for $E(s)$ and $K(s)$, depending on $s, n, \delta$ and the initial condition $x(0)$, has been derived in [98].

In practice, the assumption (c) restricting the interactions among the agents to be bidirectional (or reciprocal) often fails. The dynamics of matrix products without the type-symmetry assumption is a long-standing problem in matrix analysis and nonstationary Markov chain theory [99 101]. Some extensions of Corollary 2 to the matrices without typesymmetry assumption has been reported in [102, 103]. Most of the existing convergence criteria are however confined to the case where the model (31) exhibits consensus. The following fundamental property, established in 101], shows the equivalence between the "weak" and "strong" definitions of consensus, existing in the literature and corresponding to, respectively, weak and strong ergodicity [101] of the backward matrix products $W(k) \ldots W(0)$.

\footnotetext{
${ }^{1}$ Similar to the static model (1), the elements of $p_{i} \geq 0$ can be considered as social powers of the corresponding agents [1].

${ }^{2}$ Choosing the "agreement parameter" $\rho=\min (\delta, 1 / 2)$, the conditions (a),(b) entail the inequalities (3) from 98 .
}

Proposition 3. For any sequence $\{W(k)\}$, the following two conditions are equivalent:

1. for any $x(0)$, opinions asymptotically synchronize so that $\max _{i, j}\left|x_{i}(k)-x_{j}(k)\right| \underset{k \rightarrow \infty}{\longrightarrow} 0$;

2. for any $x(0)$, the opinions converge to a common limit $x_{i}(k) \underset{k \rightarrow \infty}{\longrightarrow} x_{*}$ (which depends on $x(0)$ ).

The following lemma gives a widely known sufficient condition for consensus [37, 91, 92, 104].

Lemma 4. Suppose that the sequence of $n \times n$ stochastic matrices $(W(k))_{k \geq 0}$ satisfies the conditions (a) and (b) from Lemma 1. Additionally, let the following repeated quasi-strong connectivity hold: there exists $T>0$ such that the following graphs

$$
\mathcal{G}_{T}(k)=\mathcal{G}[W(k)+\ldots+W(k+T-1)], \quad k \geq 0
$$

have directed spanning trees (quasi-strongly connected). Then the opinions exponentially converge to consensus $x(k) \underset{k \rightarrow \infty}{\longrightarrow} c \mathbb{1}_{n}$, where $c=c(x(0)) \in \mathbb{R}$.

The repeated quasi-strong connectivity implies that the union of each $T$ consecutive graphs is quasistrongly connected, extending thus the consensus criterion for static French-DeGroot model (11) 1, Corollary 13]. Lemmas 1 and 4 remain valid in presence of communication delays [91, 105] and can be extended to some nonlinear consensus algorithms [92, 105, 106].

\subsection{The time-varying Abelson model}

The convergence criterion, similar to Lemma 1, holds also for the time-varying counterpart of (2)

$$
\dot{x}(t)=-L[A(t)] x(t), \quad t \geq 0 .
$$

Here $A(t)=\left(a_{i j}(t)\right)$ is a non-negative matrix, whose entries are suppose to be locally $L_{1}$-summable. Unlike the static Abelson model with $A(t) \equiv A$, where opinions always converge [1], the convergence of (8) is a non-trivial problem. For the case of bidirectional (reciprocal) interactions, however, the following elegant result has been obtained in [103, 107].

Lemma 5. Suppose that the gains $a_{i j}(t)$ satisfy the following type-symmetry condition

$$
K^{-1} a_{j i}(t) \leq a_{i j}(t) \leq K a_{j i}(t) \quad \forall t \geq 0,
$$

where $K \geq 1$ is a constant. Then the functions $\dot{x}_{j}, a_{i j}\left(x_{j}-x_{i}\right)$ are $L_{1}$-summable for any $i, j$. In particular, the limit $\bar{x}=\lim _{k \rightarrow \infty} x(k)$ exists and if agents $i$ and $j$ interact persistently in the sense that

$$
\int_{0}^{\infty} a_{i j}(t) d t=\infty
$$


then their final opinions are coincident $\bar{x}_{i}=\bar{x}_{j}$.

The proofs in [103, 107] are based on the properties of the ordering permutation $y_{i}(t)=x_{j_{i}(t)}(t)$, sorting the opinions in the ascending order, we do not include them as they require some non-trivial mathematical tools. In the case where consensus is established (the graph of persistent interactions is connected), an alternative proof has been proposed in [108]. In fact, the type-symmetry assumption (59) can be replaced by the weaker cut-balance condition [103], which has recently been further relaxed to a non-instantaneous (integral) reciprocity [109]. Lemma 5 can be also extended to one-sided differential inequalities [110].

Similar to the discrete-time case 3 , the convergence of (8) without reciprocity assumptions remains a nontrivial problem. The existing convergence criteria are mainly confined to the case where consensus of opinions is established. The most general of such criteria is a continuous-time counterpart of Lemma 4, establishing exponential convergence to consensus for uniformly quasi-strong connected (UQSC) graphs.

Lemma 6. Let $A(\cdot)$ be bounded $0 \leq a_{i j}(t) \leq M$ and there exist $\varepsilon, T>0$ such that the following graphs

$$
\mathcal{G}_{\varepsilon, T}(t)=\mathcal{G}\left[\int_{t}^{t+T} A(s) d s\right], \quad t \geq 0
$$

are quasi-strongly connected for any $t \geq 0$. Then the opinions in (8) exponentially converge to consensus.

Unfortunately, a complete proof of Lemma [6 is not easily available in the literature. Most of the proofs require extra assumptions, e.g. the existence of a common root node in all the graphs $\mathcal{G}_{\varepsilon, T}$ [111], piecewise-constantness of $A(t)$ [37, 112, 113] or at least its continuity almost everywhere [108]. Analysis of the proofs in 108, 112, 113] reveals, however, the possibility to discard these additional restrictions.

The consensus criterion from Lemma 6 can be extended to some nonlinear consensus continuous-time algorithms [112 116] and retains its validity in presence of communication delays [113, 117], whereas the validity of Lemma 5 in presence of communication delays seems to be a non-trivial open problem.

Whereas the uniform connectivity from Lemma 6 is "almost" necessary for consensus (being necessary

\footnotetext{
${ }^{3}$ As can be shown [37, Lemma 2.27], if $A(t)$ is piecewiseconstant, attains values in some compact set of matrices and its consecutive switchings are separated by a positive dwell time, the model (8) in fact reduces to the discrete-time model (3).
}

for exponential consensus [112] and the consensus' robustness [108]), it is only sufficient for the convergence of opinions in the time-varying Abelson model (8) (for instance, we have seen [1] that for $A(t) \equiv A$ the opinions always converge, whereas consensus requires the quasi-strong connectivity of the graph $\mathcal{G}[A])$. At the same time, is can be easily shown that, similar to the discrete-time model, solutions always remain bounded since the convex hull spanned by the opinions does not expand [103, 107, 112, 113].

\section{Opinion dynamics with bounded confidence}

The well-known adage "birds of a feather flock together" prominently manifests the principle of homophily [118]: similar individuals interact more often and intensively than dissimilar people. Distancing from the members of other social groups, e.g. rejection of cultural forms they like [119], is an important factor of social segregation and cleavage. Humans readily assimilate opinions of like-minded individuals, accepting dissimilar opinions with discretion [120].

The idea to introduce homophily into the dynamics of opinion ("attitude") formation has in fact been proposed by Abelson [40] who first realized that the time-varying model (8) can reflect the effects of biased assimilation. As stated in [40], the variability of the "contact rates" $a_{i j}(t)$ can express that people tend to locomote into groups that share their attitudes and out of groups that do not agree with them. The latter phenomenon lies in the heart of many mathematical models, proposed recently and dealing with modifications of the French-DeGroot and Abelson models, where the influence of agent $j$ on agent $i$ is the stronger, the closer are opinions of the agents [121]. The latter principle is prominently illustrated by bounded confidence models, attracting enormous attention of a broad research community, from systems and control theorists to statistical physicists and data scientists.

Bounded confidence models stipulate that individuals are totally insensitive to opinions, falling outside their confidence sets. Simple yet instructive models of this type were independently proposed by Krause [122] and Deffuant and Weisbuch [123]. The Deffuant-Weisbuch model, based on the idea of gossiping, will be discussed in Section 5. In this section, we are primarily concerned with the model from [122], which is nowadays referred to as the HegselmannKrause (HK) and has become widely known after the 
publication of the work [124]. Along with the HK model, some of its recent extensions are considered.

\subsection{The original HK model}

We start with the original model from Krause's paper [122]. Being an extension of the French-DeGroot model (1), the HK model deals with $n$ agents, whose real opinions $x_{i} \in \mathbb{R}$ constitute the opinion vector $x=\left(x_{1}, \ldots, x_{n}\right) \in \mathbb{R}^{n}$. We introduce the fixed range of confidence $d>0$ and call the closed 4 set $\left[x_{i}-d, x_{i}+d\right] \subset \mathbb{R}$ confidence interval of agent $i$. Each agent $i$ ignores the opinions beyond his/her confidence interval, interacting only with a group of trusted individuals $I_{i}(x)=\left\{j:\left|x_{j}-x_{i}\right| \leq d\right\} \ni\{i\}$. Using $\left|I_{i}(x)\right|$ to denote their number, the $i$ th agent's opinion evolves at each step as follows

$$
x_{i}(k+1)=\frac{1}{\left|I_{i}(x(k))\right|} \sum_{j \in I_{i}(x(k))} x_{j}(k), i \in 1: n .
$$

The opinion formation process (11) is a nonlinear autonomous (time-invariant) discrete-time system

$$
\begin{gathered}
x(k+1)=\mathcal{C}(x(k)) \in \mathbb{R}^{n}, \\
\mathcal{C}(x)=\left(\mathcal{C}_{1}(x), \ldots, \mathcal{C}_{n}(x)\right)^{\top}, \\
C_{i}(x)=\frac{1}{\left|I_{i}(x)\right|} \sum_{j \in I_{i}(x)} x_{j}, \quad i \in 1: n .
\end{gathered}
$$

We refer the mapping $\mathcal{C}: \mathbb{R}^{n} \rightarrow \mathbb{R}^{n}$ to as the $H K$ operator. On the other hand, this system can be considered as the time-varying French-DeGroot model (3) with the state-dependent matrix $W(x(k))$, where

$$
W(x)=\left(w_{i j}(x)\right), \quad w_{i j}(x)= \begin{cases}1 /\left|I_{i}(x)\right|, & j \in I_{i}(x) \\ 0, & \text { otherwise. }\end{cases}
$$

Introducing the corresponding influence graph

$$
\begin{gathered}
\mathcal{G}(x)=(V, E(x), W(x)), \\
(i, j) \in E(x) \Longleftrightarrow j \in I_{i}(x) \Longleftrightarrow\left|x_{i}-x_{j}\right| \leq d,
\end{gathered}
$$

one notices that the HK model stipulates the same mechanism of the opinion formation as the original French model [76], considered in the first part [1]. Each agent updates its opinion to the average opinion of its neighbors in the influence graph. The crucial

\footnotetext{
${ }^{4}$ As will be shown in the next subsection, most properties of the HK model remain valid, replacing closed confidence intervals by open ones $\left(x_{i}-d, x_{i}+d\right)$, considered e.g. in [69].
}

difference with the French model is that this graph $\mathcal{G}(x)$ coevolves with the opinions, depending on their mutual distances 5 . This graph may lose its connectivity, leading to disagreement of the opinions.

Dynamic networks, where the nodes and topologies have mutually dependent (coevolutionary) dynamics are actively studied by physicists [125]. The HK model and its modifications constitute one important class of such networks, thoroughly studied in control theory. In the literature, one can find many other examples of coevolutionary networks, e.g. the seminal Vicsek model of phase transitions [74], multiagent models of flocks [126 128] and robotic networks with range-restricted interactions [129].

In Fig. 1, we simulate the dynamics of $n=100$ opinions for different confidence ranges $d$. The initial values $x_{i}(0)$ (same for all six experiments) are uniformly distributed on $[0,1]$. The simulation reveals some counter-intuitive phenomena, for instance, a non-monotone dependence between $d$, the number of clusters and the termination time. One could expect that the number of clusters is declining and the convergence time is decreasing as $d$ is growing. In reality, an increase in $d$ can increase the number of clusters (see the plots (a),(b) and (c),(d) in Fig. 1). There is no obvious dependence between $d$ and the convergence time, furthermore, for $d=0.25$ the convergence is visibly slower than for small $d$ (Fig. 1f) (this phenomenon of "abnormally" slow convergence to consensus has been reported in [102]; the relevant consensus states are referred in [102] as "metastable"). In Table 1, the results are compared with the prediction of a so-called $2 R$-conjecture [130, 131], stating that for the initial opinions, uniformly sampled from $[0,1]$ and $d=R<1 / 2$, the opinions converge to $\approx 1 /(2 R)$ clusters, separated by distances of $\approx 2 R$.

A natural question arises whether opinions in the HK model converge, as suggested by Fig. 1, or can oscillate for some $x(0)$. The following result, first proved in [132], shows that the HK model always converges to a fixed point in a finite number of steps.

Theorem 7. For any initial condition $x(0)$, the $H K$ dynamics (11) terminates in a finite number of steps

\footnotetext{
${ }^{5}$ The influence, or interaction graph should not be confused with the communication (information) graph, determining the agents' awareness of each other's opinions. The HK model assumes implicitly that agents are able to compute their sets $I_{i}(x(k))$, having thus the full information about the state vector $x(k)$. In this sense, the original HK dynamics (11) unfolds over a social network with all-to-all communication.
} 


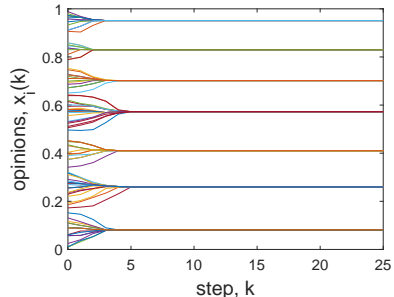

(a) $d=0.05$

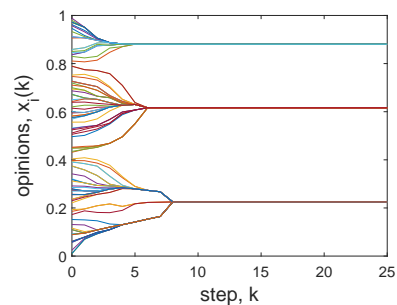

(c) $d=0.11$

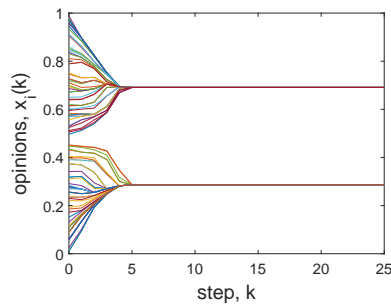

(e) $d=0.2$

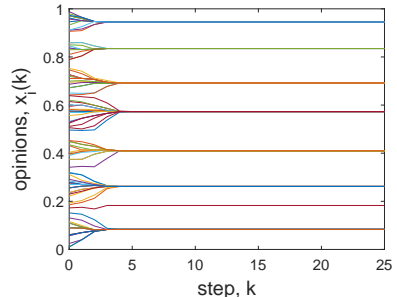

(b) $d=0.06$

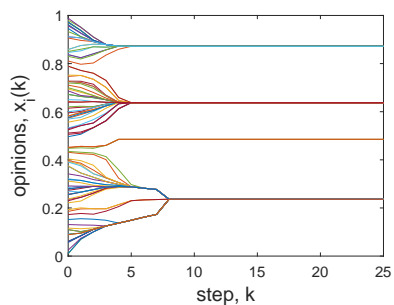

(d) $d=0.12$

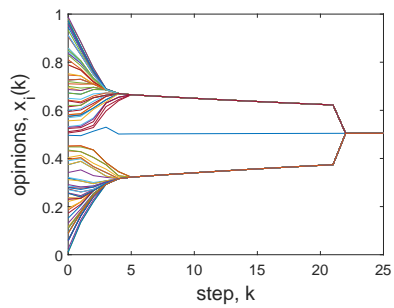

(f) $d=0.25$
Figure 1: The HK model with $n=100$ agents and different $d$.

$x(k) \equiv \bar{x} \forall k \geq k_{*}$, where the final opinion $\bar{x}$ and the termination time depend on $x(0)$ and $d$. After the model's termination, any agents $i, j$ either are in consensus $\bar{x}_{i}=\bar{x}_{j}$ or distrust each other $\left|\bar{x}_{i}-\bar{x}_{j}\right|>d$.

Note that Theorem 7 provides no information about stability of equilibria points. In general, solutions of the HK model can converge to unstable equilibria, however, experiments show that for randomly chosen initial opinions such a behavior is untypical [69]. For criteria of (local) asymptotic stability we refer the reader to $[69$.

There are several ways to prove Theorem 7 . The original proof [132] extends the ideas from [122] and is based on the matrix products convergence. Another approach is based on Lyapunov energy-like functions. The relevant methods will be discussed in the next subsections, dealing with multidimensional extensions the HK model. The proof we outline in this subsection is based on the important orderpreservation property of the HK model (11) and gives the best known estimate for its termination time.

Lemma 8. [12] The HK operator $\mathcal{C}$ from (12) pre-
Table 1: Actual numbers of clusters vs. $2 R$-conjecture 130]

\begin{tabular}{c|c|c}
\hline \hline$d$ & Number of clusters & Rounded $1 /(2 d)$ \\
\hline 0.05 & 7 & 10 \\
0.06 & 8 & 8 \\
0.11 & 3 & 5 \\
0.12 & 4 & 4 \\
0.2 & 2 & 3 \\
0.25 & 1 & 2 \\
\hline
\end{tabular}

serves the order of the elements $x_{1}, \ldots, x_{n}$, that is, if $j_{1}, \ldots, j_{n}$ is the ordering permutation of indices $x_{j_{1}} \leq \ldots \leq x_{j_{n}}$, then $\mathcal{C}_{j_{1}}(x) \leq \ldots \leq \mathcal{C}_{j_{n}}(x)$.

Lemma 8 can be proved via induction on $n$. For $n=1$, the statement is obvious. Assuming that it holds for the HK operator of dimension $n-1$, denoted $\tilde{\mathcal{C}}: \mathbb{R}^{n-1} \rightarrow \mathbb{R}^{n-1}$, our goal is to prove it for the HK operator $\mathcal{C}: \mathbb{R}^{n} \rightarrow \mathbb{R}^{n}$. It suffices to consider the case where $x$ is sorted in the ascending order $x_{1} \leq$ $\ldots \leq x_{n}$, i.e. $j_{i}=i$. If $x_{n}-x_{1} \leq d$, then $\mathcal{C}_{1}(x)=$ $\ldots=\mathcal{C}_{n}(x)=\left(x_{1}+\ldots+x_{n}\right) / n$, and the statement is obvious. Otherwise, let $j=\min I_{n}(x)<n$ and $l=\max I_{1}(x)>1$ and $x^{h}=\left(x_{1}, \ldots, x_{n-1}\right)^{\top}, x^{t}=$ $\left(x_{2}, \ldots, x_{n}\right)^{\top}$ be the "head" and "tail" truncations of $x$. For $i \leq j$ one has $x_{i} \leq x_{j}<x_{n}-d$, that is, agent $i$ is not influenced by agent $n$, and thus $\tilde{\mathcal{C}}_{i}\left(x^{h}\right)=\mathcal{C}_{i}(x)$. For the same reason, $\tilde{\mathcal{C}}_{i}\left(x^{t}\right)=\mathcal{C}_{i}(x)$ whenever $i \geq l$. Therefore, the sequences $\left\{C_{i}(x)\right\}_{i=1}^{j}$ and $\left\{C_{i}(x)\right\}_{i=l}^{n}$ are non-decreasing. If $l \leq j$, the induction step is proved. Assuming that $j<l$, we have to show that

$$
C_{j}(x) \leq C_{r}(x) \leq C_{s}(x) \leq C_{l}(x) .
$$

whenever $j<r<s<l$. Since $r, s \in I_{1}(x) \cap I_{n}(x)$,

$$
\begin{gathered}
C_{r}(x) \stackrel{(*)}{=} \frac{m_{r} \tilde{C}_{r}\left(x^{h}\right)+x_{n}}{m_{r}+1}=\frac{m_{r} \tilde{C}_{r}\left(x^{t}\right)+x_{1}}{m_{r}+1}, \\
C_{s}(x) \stackrel{(+)}{=} \frac{m_{s} \tilde{C}_{s}\left(x^{h}\right)+x_{n}}{m_{s}+1} \stackrel{(!)}{=} \frac{m_{s} \tilde{C}_{s}\left(x^{t}\right)+x_{1}}{m_{s}+1},
\end{gathered}
$$

where $m_{r}=\left|I_{r}(x)\right|-1$ and $m_{s}=\left|I_{s}(x)\right|-1$. Recalling that $\tilde{C}_{r}\left(x^{h}\right) \geq \tilde{C}_{j}\left(x^{h}\right)=\mathcal{C}_{j}(x)$ and $x_{n}=\max _{i} x_{i} \geq$ $\tilde{C}_{j}\left(x^{h}\right)$, one proves the leftmost inequality in (14) by using the equality $(*)$ from (15). Similarly, the equality (!) entails the rightmost inequality in (14). Using $\left(^{*}\right)$ and $(+)$, the mid inequality in (14) shapes into

$$
m_{r} \tilde{C}_{r}\left(x^{h}\right) \leq m_{s} \tilde{C}_{s}\left(x^{h}\right)+\left(m_{r}-m_{s}\right) x_{n} .
$$

To prove the latter inequality, note that $\tilde{C}_{s}\left(x^{h}\right) \geq$ $\tilde{C}_{r}\left(x^{h}\right)$ by assumption, $x_{n}=\max _{i} x_{i} \geq \tilde{C}_{r}\left(x^{h}\right)$ and 
$I_{s}(x) \subseteq I_{r}(x)$, entailing that $m_{r} \geq m_{s}$. This finishes the proof of (14) and of the induction step.

From now on until the end of this subsection, the agents' opinions are numbered in the ascending order

$$
x_{1} \leq \ldots \leq x_{n}
$$

If the initial vector of opinions $x(0)$ is sorted as in (16), this order of opinions is preserved at any iteration due to Lemma 8 . We say that the opinions $\left(x_{i}, \ldots, x_{m}\right)$ constitute a $d$-chain [132] if the distances between consecutive opinions $x_{i+1}-x_{j}, \ldots, x_{m}-x_{m-1}$ are $\leq d$, that is, the graph $\mathcal{G}(x)$ from (13) contains a chain of $\operatorname{arcs} i \leftrightarrow i+1 \leftrightarrow \ldots \leftrightarrow i+m$. Obviously, the vector of opinions $x$ consists of several maximal $d$ chains (that are not contained by any longer $d$-chain), which correspond to the connected components of the graph $\mathcal{G}(x)$. This is illustrated in Fig. 2, where the opinions split into three maximal $d$-chains $\left(x_{1}, x_{2}\right)$, $\left(x_{3}, x_{4}\right)$ and $\left(x_{5}, x_{6}, x_{7}\right)$, standing for the three connected components of the graph $\mathcal{G}(x)$.

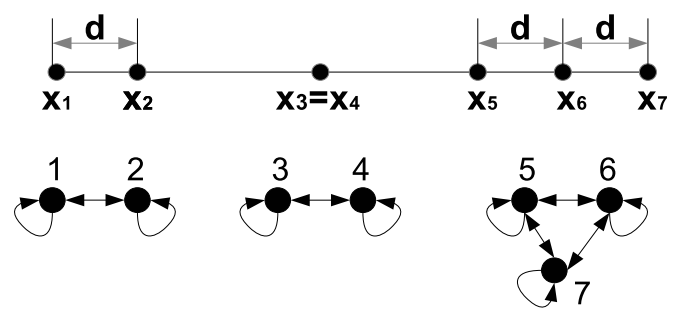

Figure 2: Opinions of $n=7$ agents and the graph $\mathcal{G}(x)$

It can be easily shown that two different maximal $d$-chains can never merge, and the corresponding sets of agents do not influence each other at any step.

Lemma 9. Suppose that the initial opinions $x_{i}(0)$ are sorted in the ascending order (16) and $x_{i+1}(0)-$ $x_{i}(0)>d$. Then $x_{i+1}(k)$ is non-decreasing and $x_{i}(k)$ is non-increasing in $k$, and thus $x_{i+1}(k)-x_{i}(k)>d$. In other words, two maximal d-chains cannot merge.

Proof. We will show via induction on $k$ that

$$
x_{i}(k+1) \leq x_{i}(k) \leq x_{i+1}(k)-d \leq x_{i+1}(k+1)-d .
$$

We prove the induction base $k=0$. Agent $i$ can interact at step $k=0$ only with some of agents $1, \ldots, i-1$, and thus $x_{i}(1) \leq x_{i}(0)=\max _{j \leq i} x_{j}(0)$. Similarly, agent $i+1$ can interact only with agents $i+2, \ldots, n$, so that $x_{i+1}(1) \geq x_{i+1}(0)$. This proves (17) for $k=0$. The step from $k$ to $k+1$ is proved in the same way.
Corollary 10. If two agents $i$ and $j$ belong to different connected components of $\mathcal{G}\left(x\left(k_{0}\right)\right)$, there are no walks connecting them in any of the graphs $\mathcal{G}(x(k))$, $k \geq k_{0}$. As $k$ grows, the strong components of $\mathcal{G}(x(k))$ can split into smaller components but cannot merge.

Lemma 9 can also be reformulated as follows: in each maximal $d$-chain $x_{j}(k) \leq \ldots \leq x_{m}(k)$ the leftmost opinion $x_{j}(k)$ is non-decreasing $x_{j}(k+$ $1) \geq x_{j}(k)$, whereas the rightmost opinion is nonincreasing $x_{m}(k+1) \leq x_{m}(k)$. In particular, the $d i$ ameter of the $d$-chain $x_{m}(k)-x_{j}(k)$ is non-increasing.

If the diameter of a maximal $d$-chain is not greater than $d$, at the next step this chain collapses into a group of identical opinions $x_{i}=x_{i+1}=\ldots=x_{i+m}$, which we henceforth refer to as a $x_{i}=x_{i+1}=$ $\ldots=x_{i+m}$ (an example of such a cluster is the pair of opinions $x_{3}, x_{4}$ in Fig. 2). This happens e.g. with the maximal $d$-chain with only two opinions $x_{i}(k)<x_{i+1}(k)$. Maximal $d$-chains containing 3 or 4 opinions in fact also collapse into consensus clusters after, respectively, 2 and 5 steps [122], and hence the HK model with $n<5$ agents always converges to consensus. This statement does not hold for $n>5$ : maximal $d$-chains with 5 and more opinions can split into shorter $d$-chains, which in turn can further split or converge to different consensus clusters. For this reason, the HK model with $n \geq 5$ agents may fail to reach consensus even when $\mathcal{G}(x(0))$ is connected.

A more accurate analysis of $d$-chains reveals the following important property [133].

Lemma 11. During each two consecutive steps $k$ and $k+1$, any maximal chain in the vector $x(k)$ collapses into a singleton, splits into several maximal $d$-chains or reduces in diameter by at least $d /\left(n^{2}\right)$.

There can be at most $n-1$ times $k$ at which one of the chains collapses, and at most $n-1$ splitting times. Obviously, the sum of the diameters of all $d$ chains is not greater than $(n-1) d$, so the diameter can be decreased no more than $(n-1) n^{2}$ times. Hence, the HK dynamics terminates in no more than $k_{*} \leq$ $2\left((n-1) n^{2}+2(n-1)\right)=2 n^{3}-2(n-1)^{2}$ steps.

Corollary 12. [133] The HK model with $n$ agents terminates in no more that $O\left(n^{3}\right)$ steps.

The polynomial convergence time has been first conjectured in [98], where the HK model has been proved to terminate in $n^{O(n)}$ steps. An alternative proof of Corollary 12, based on Lyapunov analysis, 
has been given in [134] (with the upper bound of termination time $3 n^{3}+n$ ). More conservative polynomial estimates for the termination time have been obtained in [129, 135, 136]; the approaches developed there are also applicable to more general multidimensional HK models, considered in the next subsection. Notice that Lemma 11] gives only an upper bound of the convergence time. To the best of the authors' knowledge, the tightness of this estimate remains a non-trivial open problem. For some initial conditions the HK model terminates in $\Omega\left(n^{2}\right)$ steps [137].

\subsection{The multidimensional HK model}

A natural extension of the HK model deals with multidimensional opinions $x_{i}(k) \in \mathbb{R}^{m}$ [138]. Choosing some norm $\|\cdot\|$ on $\mathbb{R}^{m}$, the confidence interval for agent $i$ is replaced by the ball $\left\{\xi \in \mathbb{R}^{m}:\left\|\xi-x_{i}\right\| \leq d\right\}$ and hence the set of trusted individuals is defined as

$$
I_{i}(x)=\left\{j:\left\|x_{j}-x_{i}\right\| \leq d\right\},
$$

where $x \in \mathbb{R}^{n m}$ denotes the column vector, obtained by stacking $x_{1}, \ldots, x_{n} \in \mathbb{R}^{m}$ on top of each other. Here $\|\cdot\|$ can be an arbitrary norm on $\mathbb{R}^{m}$, however most of the existing works [133, 135, 138 140] deal with the Euclidean norm $\|\xi\|=\sqrt{\xi^{\top} \xi}$.

Considering the scalar elements of the multidimensional opinions as individual's positions on different issues, the definition of trust sets (18) imposes an implicit dependence between these issues. In particular, two individuals $i, j$ that strongly disagree on the $s$ th issue (e.g. $\left|x_{i, s}-x_{j, s}\right| \geq d$ ) ignore each other's positions on all remaining issues since $i \notin I_{j}(x), j \notin I_{i}(x)$.

Unlike the scalar case, for $m>1$ the connected components of the graph $\mathcal{G}(x)$ can not only split, but also merge as shown in Fig. 3. Consider $n=4$ opinion vectors $x_{i}(0) \in \mathbb{R}^{3}$, being the vertices of a tetrahedron $x_{1}(0)=(0,0, b), x_{2}(0)=(0,0,-b)$, $x_{3}(0)=(a, 0,0), x_{4}(0)=(0, a, 0)$, where $0<b<d / 2$ and $\sqrt{d^{2}-b^{2}}<a \leq d$. It can be easily shown that the graph $\mathcal{G}(x(0))$ has three connected components (Fig. 3r) since $I_{1}(x)=I_{2}(x)=\{1,2\}$ and $I_{i}(x)=\{i\}$ for $i=3,4$. At the next step (Fig. 3b) one has

$$
x_{1}(1)=x_{2}(1)=(0,0,0), x_{3}(1)=x_{3}(0), x_{4}(1)=x_{4}(0),
$$

and thus the graph $\mathcal{G}(x(1))$ is connected (Fig. 3d) (in fact, agents reach consensus in $k=3$ steps).

A natural question thus arises whether result of Theorem 7 holds for the multidimensional HK model (11), (18), that is, the dynamics terminate in

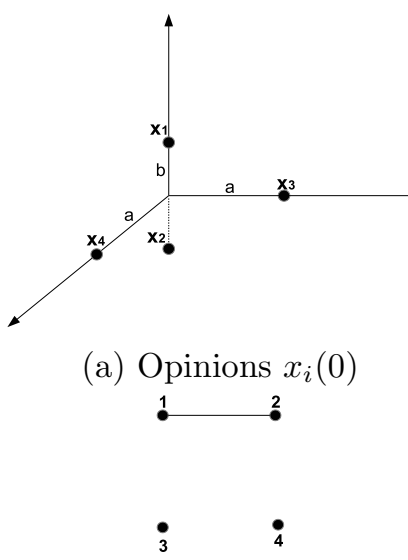

(c) The graph $\mathcal{G}(x(0))$ (b) Opinions $x_{i}(1)$

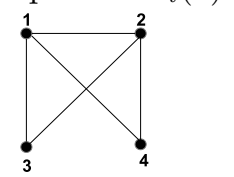

(d) The graph $\mathcal{G}(x(1))$

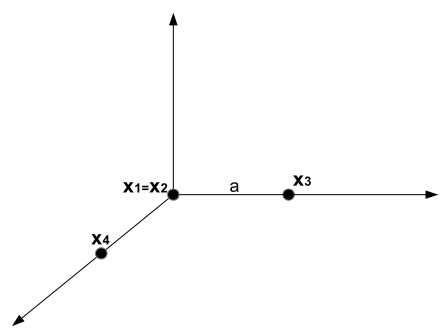

Figure 3: Two connected components of $\mathcal{G}(x)$ merge

finite time. An affirmative answer is giving by the following theorem.

Theorem 13. For any choice of the norm $\|\cdot\|$, the model (11),(18) terminates in finite number of steps.

The simplest way to prove Theorem 13 is to apply Lemma 1. It can be easily shown that the HK dynamics (11), (18) can be written as the time-varying French-DeGroot model (3), where the stochastic matrix $W(k)=W(x(k))$ is state-dependent and satisfies all assumptions of Lemma 1. Hence the opinions converge, and it remains to prove finite-time convergence. Considering the graph of persistent interactions $\mathcal{G}_{\infty}$ corresponding to some specific solution. As we have noticed, condition (a) in Lemma 1 implies that if nodes $i$ and $j$ are not connected in $\mathcal{G}_{\infty}$, they are not connected in $\mathcal{G}(x(k))$ for large $k$. Hence for large $k$ the nodes from different components of $\mathcal{G}_{\infty}$ do not influence each other. Consider now connected components $\mathcal{G}_{\infty}^{s}=\left(V^{s}, E_{\infty}^{s}\right)$ of the graph $\mathcal{G}_{\infty}$. Thanks to Lemma1, in each component consensus is established

$$
x_{i}(k) \underset{k \rightarrow \infty}{\longrightarrow} \bar{x}^{s} \quad \forall i \in V^{s} .
$$

Therefore, for large $k$ one has $\left|x_{i}(k)-x_{j}(k)\right|<$ $d \forall i, j \in V^{s}$, and hence $I_{i}(x)=V^{s} \forall i \in V^{s}$. This means that at some step $k=k_{0}$ the opinions in each connected component of $\mathcal{G}(x(k))$ become equal

$$
x_{i}\left(k_{0}+1\right)=\frac{1}{\left|V^{s}\right|} \sum_{j \in V^{s}} x_{j}\left(k_{0}\right) \quad \forall i \in V^{s},
$$

that is, the HK model terminates in finite time.

A natural question arises whether the termination time depends on the number of agents $n$ polynomially. The positive answer has been given in $[133,135$, 
139, 141]. In the Euclidean norm, the best existing estimate for the dimensions $m \geq 2$ seems to be $2 n^{4}$ steps [140]. This and other existing estimates for the convergence time of the multidimensional HK model are based on special Lyapunov functions that will be discussed in the next subsection. It is remarkable that the best known lower bound for the dimensions $m \geq 2$ is $\Omega\left(n^{2}\right)$ (same as in one-dimensional case); as demonstrated in [133], the agents placed in the vertices of regular (planar) $n$-gon with the side $d$ reach consensus in no less than $n^{2} / 28$ steps.

It can be noticed that Theorem 13 remains valid for more general distance-based models of opinion formation, examined in [142, 143]. Given a function $\varphi:[0, \infty) \rightarrow[0, \infty)$, the multidimensional opinion vectors $x_{i}(k) \in \mathbb{R}^{m}$ evolve as follows

$$
\begin{gathered}
x_{i}(k+1)=\frac{\sum_{j=1}^{n} \varphi_{i j}(k) x_{j}(k)}{\sum_{j=1}^{N} \varphi_{i j}(k)}, \\
\varphi_{i j}(k):=\varphi\left(\left|x_{j}(k)-x_{i}(k)\right|^{2}\right) .
\end{gathered}
$$

Obviously, the multidimensional HK model with Euclidean norm is a special case of the model (19), where $\varphi$ stands for the indicator function of the interval $\left[0, d^{2}\right]$. In [143], another function was considered

$$
\varphi(\sigma)= \begin{cases}a, & \sigma \leq d_{1}^{2} \\ b, & \sigma \in\left(d_{1}^{2}, d_{2}^{2}\right), \\ 0, & \sigma>d_{2}^{2},\end{cases}
$$

where $0<a<b$ and $0<d_{1}<d_{2}$. This function $\varphi(\sigma)$ represents the phenomenon of heterophily: moderately distant opinions are attracted more intensively than similar ones. A counter-intuitive phenomenon, reported in [143], is the facilitation of consensus by these "heterophilous" interactions.

The result of Theorem 13 (except for the finite-time convergence) can be easily extended to systems (19) with special functions $\varphi(\cdot)$.

Lemma 14. Suppose that $\varphi(\sigma) \in\{0\} \cup[a, b] \forall \sigma \geq$ 0 for some constants $0<a<b$ and, furthermore, $\varphi(0)>0$. Then the opinions, obeying (19), converge (yet do not stabilize after finite number of steps).

Lemma 14 easily follows from Lemma 1. As will be discussed in the next subsection, in fact the system (19) converges for many other functions $\varphi(\cdot)$, including continuous ones; the relevant convergence results are based on special Lyapunov functions. As a further extension of (19), one may consider a model where each pair of agents $(i, j)$ is endowed with its own distance-measuring function $\varphi^{i j}(\sigma)$; without loss of generality we assume that $\varphi^{i i}(\sigma) \equiv \varphi^{i i}>0$.

$$
\begin{aligned}
& x_{i}(k+1)=\frac{\sum_{j=1}^{n} \varphi_{i j}(k) x_{j}(k)}{\sum_{j=1}^{N} \varphi_{i j}(k)}, \\
& \varphi_{i j}(k):=\varphi^{i j}\left(\left|x_{j}(k)-x_{i}(k)\right|^{2}\right) .
\end{aligned}
$$

Obviously, if $\varphi^{i j}(\sigma) \in\{0\} \cup[a, b]$ for some $0<a<b$ and $\varphi^{i j}(\sigma)>0 \Leftrightarrow \varphi^{j i}(\sigma)>0$, the result of Lemma 14 remains valid for the heterogeneous model (20). A special case of model (20) has been proposed in 69], choosing the mappings $\varphi^{i j}$ as follows

$$
\varphi^{i j}(\sigma)= \begin{cases}w_{j}, & \sigma<d^{2} \\ 0, & \sigma \geq d^{2} .\end{cases}
$$

Here $w_{1}, \ldots, w_{n}>0$ are positive "weights" or "reputations" [144] of the agents. Similarly to the usual HK model, in the scalar case $(m=1)$ such a model provides the order preservation of the opinions [69].

\subsection{Lyapunov methods for the HK model}

Henceforth the "term HK model" stands for the multidimensional model (11), (18) with the Euclidean norm $\|x\|=|x|$.

The standard Lyapunov function used to study consensus algorithms (3), (8) is the diameter of the convex hull, spanned by the agents' opinions [92, 112]. This Lyapunov function, however, appears to be most useful in the case where consensus of opinions is established (and thus their convex hull collapses into a singleton), whereas the opinions in the HK model, in general, split into several clusters. The special structure of the HK model (11), however, implies the existence of another piecewise-smooth Lyapunov function

$$
\mathcal{E}(x) \triangleq \sum_{i, j=1}^{n} \min \left(\left|x_{i}-x_{j}\right|^{2}, d^{2}\right) .
$$

This "energy" function is a special case of more general Lyapunov functions, proposed in the seminal paper [145] to examine some types of nonlinear consensus algorithms (a similar Lyapunov function has been also used to study "continuum" bounded confidence opinion dynamics in [130]). The function (21) proves to be non-increasing along the system's trajectories; moreover, it strictly decreases until the opinion evolution terminates, as implied by the following. 
Proposition 15. [133, 145] For any solution of the $H K$ model and $k=0,1, \ldots$, the inequality holds

$$
\mathcal{E}(x(k))-\mathcal{E}(x(k+1)) \geq 4 \sum_{i=1}^{n}\left|x_{i}(k+1)-x_{i}(k)\right|^{2} .
$$

The inequality (22) implies the following bound for kinetic 2-energy (defined in (6) ) of the HK model

$$
K(2) \leq \mathcal{E}(x(0)) \leq d^{2} n(n-1) .
$$

Using elegant techniques from algebraic graph theory, it has been shown in [140] that during each step of the opinion formation either two opinions merge or the energy $\mathcal{E}(x(k))$ is decreased by at least $d /\left(2 n^{2}\right)$, and hence the HK dynamics terminates in $O\left(n^{4}\right)$ steps independent of the opinions' dimension $m$.

Proposition (15) can be extended to a more general model (19) with a non-increasing function $\varphi(\cdot)$.

Lemma 16. [142] Suppose that $\varphi(\cdot)$ in (19) is nonincreasing. Then the "energy" function

$$
\mathcal{E}_{\varphi}(x)=\sum_{i, j=1}^{n} \Phi\left(\left|x_{i}-x_{j}\right|^{2}\right), \Phi(r)=\int_{0}^{r} \varphi(\sigma) d \sigma,
$$

is non-increasing and satisfies the inequality

$$
\begin{aligned}
& \mathcal{E}_{\varphi}(x(k))-\mathcal{E}_{\varphi}(x(k+1)) \geq \\
& \quad \geq \sum_{i, j=1}^{n} \varphi_{i j}(k)\left|\Delta x_{i}(k)+\Delta x_{j}(k)\right|^{2} \\
& \quad \geq 4 \varphi(0) \sum_{i=1}^{n}\left|\Delta x_{i}(k)\right|^{2},
\end{aligned}
$$

where $\Delta x_{i}(k)=x_{i}(k+1)-x_{i}(k)$.

Obviously, Lemma 16 implies Proposition 15 since in the case of the usual HK model one has $\Phi(r)=$ $\min \left(r, d^{2}\right)$ and $\varphi(0)=1$. We give the sketch of the proof of Lemma 16, presented 6 in [142] (Proposition 4.1). The proof is based on the following three relations. First, the function $\Phi(r)$ is concave $\left(\Phi^{\prime}(r)=\varphi(r)\right.$ is non-increasing $)$ and hence

$$
\Phi(a)-\Phi(b) \geq \varphi(a)(a-b) \quad \forall a, b \geq 0 .
$$

\footnotetext{
${ }^{6}$ In fact, Proposition 4.1 in 142] reports a stronger inequality $\mathcal{E}(x(k))-\mathcal{E}(x(k+1)) \geq 4 \sum_{i, j} \varphi_{i j}(k)\left|\Delta x_{i}(k)\right|^{2}$ whose proof seems to be elusive: the latter inequality relies on (25) and the incorrect inequality $-\sum_{i, j} \varphi_{i j}(k)\left|\Delta x_{i}(k)+\Delta x_{j}(k)\right|^{2} \leq$ $-4 \sum_{i, j} \varphi_{i j}(k)\left|\Delta x_{i}(k)\right|^{2}$ (which holds with $\geq$ instead of $\leq$ ).
}

Second, $\varphi_{i j}(k)=\varphi_{j i}(k)$, and therefore

$$
-2 \sum_{i, j} \varphi_{i j}(k) \xi_{i}^{\top}\left(\eta_{j}-\eta_{i}\right)=\sum_{i, j} \varphi_{i j}(k)\left(\xi_{j}-\xi_{i}\right)^{\top}\left(\eta_{j}-\eta_{i}\right)
$$

for any set of vectors $\xi_{i}, \eta_{i} \in \mathbb{R}^{m}$ (here $i=1, \ldots, n$ ). Finally, for each $i$ (19) implies the following

$$
\sum_{j=1}^{n} \varphi_{i j}(k) \Delta x_{i}(k)=\sum_{j=1}^{n} \varphi_{i j}(k)\left(x_{j}(k)-x_{i}(k)\right) .
$$

Denoting $z_{i j}(k)=x_{j}(k)-x_{i}(k)$, one obtains

$$
\begin{aligned}
& \sum_{i, j} \varphi_{i j}(k)\left(\Delta x_{j}(k)-\Delta x_{i}(k)\right)^{\top} z_{i j}(k) \stackrel{(27)}{=} \\
& =-2 \sum_{i, j} \varphi_{i j}(k) \Delta x_{i}(k)^{\top} z_{i j}(k) \stackrel{(28)}{=} \\
& =-2 \sum_{i, j} \varphi_{i j}(k)\left|\Delta x_{i}(k)\right|^{2}= \\
& =-\sum_{i, j} \varphi_{i j}(k)\left(\left|\Delta x_{j}(k)\right|^{2}+\left|\Delta x_{i}(k)\right|^{2}\right) .
\end{aligned}
$$

By noticing that $z_{i j}(k+1)=z_{i j}(k)+\Delta x_{j}(k)-\Delta x_{i}(k)$, the latter equality entails that

$$
\begin{aligned}
& \mathcal{E}_{\varphi}(x(k))-\mathcal{E}_{\varphi}(x(k+1)) \stackrel{(24),(26)}{\geq} \\
& \quad \geq \sum_{i, j} \varphi_{i j}(k)\left(\left|z_{i j}(k)\right|^{2}-\left|z_{i j}(k+1)\right|^{2}\right)= \\
& \quad=-\sum_{i, j} \varphi_{i j}(k)\left|\Delta x_{j}(k)-\Delta x_{i}(k)\right|^{2}- \\
& \quad-2 \sum_{i, j} \varphi_{i j}(k)\left(\Delta x_{j}(k)-\Delta x_{i}(k)\right)^{\top} z_{i j}(k) \stackrel{(29)}{=} \\
& \quad=\sum_{i, j} \varphi_{i j}(k)\left|\Delta x_{j}(k)+\Delta x_{i}(k)\right|^{2} \geq \\
& \quad \geq 4 \sum_{i} \varphi_{i i}(k)\left|\Delta x_{i}(k)\right|^{2}=4 \varphi(0) \sum_{i}\left|\Delta x_{i}(k)\right|^{2} . \square
\end{aligned}
$$

Using the inequality (25), it is possible to establish convergence of the model (19) when the function $\varphi(\cdot)$ does not satisfy the conditions of Lemma 14 [142, 143, 145]. The result of [142, Theorem 3] establishes convergence of the model (19) for any nonincreasing and concave function $\varphi(\cdot)$ with a compact support, on which the inequality $\left|\varphi^{\prime}(r)\right|^{2} \leq C \varphi(r)$ should hold for some constant $C>0$. The method developed in [145] allows to discard the concavity assumption [145, Corollary 1] in the scalar case.

It should be noticed that (21) is not the only Lyapunov function that can be used to examine the HK 
model. Alternative Lyapunov functions have been employed in [134, 146] and [136, 138] (the latter works deal with a special Lyapunov functional, based on the construction of a so-called adjoint system and depending on the whole trajectory of the HK model).

\subsection{Extensions and related models}

Recently a lot of alternative models based on the ideas of bounded confidence and extending the HK model in different ways, have been proposed. One extension, known as the Deffuant-Weisbuch model, will be considered in Section 5, dealing with asynchronous gossip-based models. Focused on agentbased models, this tutorial also does not address statistical (Eulerian) bounded confidence models studied in 68, 70, 71, 130, 147 149]. Some other extensions are briefly summarized in this subsection.

\subsubsection{Continuous-time bounded confidence models}

Many results, available for the original HK model, have been extended to its continuous-time counterparts. The direct extension of the scalar HK model (11), introduced in [147], is as follows

$$
\dot{x}_{i}(t)=\sum_{j:\left|x_{j}(t)-x_{i}(t)\right|<d}\left(x_{j}(t)-x_{i}(t)\right) \in \mathbb{R}
$$

(following 69], this model deals with open confidence intervals; modifications with closed intervals have been also considered [150]).

The differential equation (30) has a discontinuous right-hand side, which gives rise to the problem of solution existence. As has been shown in [147], the classical Caratheódory solution (with $x_{i}(t)$ absolutely continuous for $t \geq 0$ and, moreover, differentiable everywhere except for a countable set of points) exists for almost all initial conditions $x(0)$. Using the result of Lemma 5, it can be easily shown that every such solution converges as $t \rightarrow \infty$. In general, the solutions does not reach consensus, however, consensus has been proved in the situation where the initial interaction graph $\mathcal{G}(x(0))$ is "densely" connected [150]. Alternatively, one may consider generalized solutions (replacing, as usual, the discontinuous right-hand side by a differential inclusion). In [151], the existence of Krasovskii solutions for any initial conditions and their convergence have been shown. Krasovskii solution is not uniquely determined by its initial condition and, in general, may exhibit some "pathological" behavior (e.g. the solution starting at an equilibrim point may leave it and converge to another equilibrium). To avoid numerical instabilities, caused by the discontinuities, the following "smoothed" modification of the HK model can be introduced [150, 151]

$$
\dot{x}_{i}(t)=\sum_{j} s\left(x_{j}-x_{i}\right)\left(x_{j}(t)-x_{i}(t)\right) \in \mathbb{R},
$$

where $s: \mathbb{R} \rightarrow \mathbb{R}_{+}$stands for some even continuous function (similar multidimensional models, extending (19) to the case of continuous time, have been examined in 142, 143, 152]). Smoothed and discontinuous bounded confidence models inherit many properties of the original HK model, e.g. the order preservation property [151]. Similar to the discretetime model [69], criteria for the equilibria's local stability can be obtained [151, 152]. Unlike the discretetime model, the models (30) and (31) also preserve the "average" opinion $\bar{x}(t)=n^{-1}\left(x_{1}(t)+\ldots+x_{n}(t)\right)$ since, obviously, $\dot{\bar{x}}(t)=0$ almost everywhere.

\subsubsection{Effects of stubborness}

As has been discussed in Part I of this tutorial [1], the dynamics of the French-DeGroot model changes dramatically in presence of stubborn individuals (keeping their opinions unchanged). Further relaxation of the stubborness concept leads to the Friedkin-Johnsen (FJ) model, where some agents can be "partially" stubborn (prejudiced). Such agents assimilate the others' opinions, being at the same time "anchored" at their initial opinions and factoring them into every step of the opinion iteration. Similar extensions have been suggested for the HK model.

In their work [153], Hegselmann and Krause have proposed a model that inherits both the HK and FJ models. Consider $n$ agents with $m$-dimensional opinions $x_{1}, \ldots, x_{n} \in \mathbb{R}^{m}$ and fix one point $T \in \mathbb{R}^{m}$ in the opinion space referred to as the "truth". Assigning agent $i$ with a constant $\lambda_{i} \in[0,1]$ that characterizes the attractiveness of the truth for this agent and being a counterpart of the susceptibility in the FJ model [1], the dynamics from [153] is as follows

$$
x_{i}(t+1)=\frac{\lambda_{i}}{\left|I_{i}(x(t))\right|} \sum_{j \in I_{i}(x(t))} x_{j}+\left(1-\lambda_{i}\right) T,
$$

where the $I_{i}(x)$ stand for the sets of trusted individuals (18). As discussed in the more recent work [82], the "truth" value may be considered as some external signal, influencing the system. The agents with $1-\lambda_{i}>0$ are referred in [153] as truth seekers. In 
the absence of truth seekers, (32) boils down to the usual HK model (11). The truth seekers with $\lambda_{i}=T$ are stubborn: $x_{i}(t) \equiv T$ for $t \geq 1$. Comparing the model (32) with the FJ model, one notices two principal differences: first, the influence graph is distancedependent (giving rise to the convergence problem 7 ) and, second, the agents have equal prejudices.

The most general result, concerned with the convergence of the model (32), is as follows.

Theorem 17. [82, 98] The opinions of all truth seekers $\left(\lambda_{i}<1\right)$ converge to the truth $8 x_{i}(t) \underset{t \rightarrow \infty}{\longrightarrow} T$. The opinions of the remaining agents (with $\lambda_{i}=0$ ) either converge to $T$ or stabilize in finite time at some values $\bar{x}_{i}$ such that $\left|\bar{x}_{i}-T\right| \geq d$.

Notice that convergence of the opinions to the truth value is usually asymptotical but not finite-time, as can be easily shown for the system of $n=2$ agent, one of them being stubborn $x_{1}(t) \equiv T \in \mathbb{R}$ and the other starting at some point $x_{2}(0) \in(T-d, T+d)$. Some conservative estimates for the convergence rate have been obtained in 98]. A natural question when the opinions reach consensus at $T$ in presence of agents with $\lambda_{i}=0$ still remains open. Numerical results, reported in [82] for the special case where all agents are either stubborn $\left(\lambda_{i}=1\right)$ or do not seek the truth $\left(\lambda_{i}=0\right)$ have revealed a highly non-trivial and counter-intuitive dependence between the number of stubborn agents, the confidence bound $d$ and the number of clusters. In particular, for some $d$ consensus is reached for small number of stubborn individuals and is destroyed as their number increases.

A more general class of models with stubborn individuals have been studied in [155] by using Lyapunov techniques. In [155], the class of "inertial" HK models has been studied 9 , obeying the equations

$$
x_{i}(t+1)=\left(1-\lambda_{i}\right) x_{i}(t)+\frac{\lambda_{i}}{\left|I_{i}(x(t))\right|} \sum_{j \in I_{i}(x(t))} x_{j} .
$$

Here $\lambda_{i} \in[0,1]$ (referred in [155] to as the coefficient of inertia); the agents with $\lambda_{i}(t) \equiv 0$ are stubborn (in [155], they are called "close-minded"). For the

\footnotetext{
${ }^{7}$ In general, opinions in the FJ model with time-varying influence graph can oscillate even when the graph remains strongly connected and some agents have $\lambda_{i}<1$, see 97].

${ }^{8}$ In the scalar case, this convergence was proved in [153, 154].

${ }^{9}$ Note that the "inertial" bounded confidence models have been also introduced and numerically studied in [156, 157].
}

general system (33), extensions of Proposition (15) and the inequality (23) have been established in [155]. These results allow to prove that the HK model with stubborn agents, that is, the system (33) where each agent has either $\lambda_{i}=0$ or $\lambda_{i}=1$, always converges.

Theorem 18. [155] The opinions in the system (33) with $\lambda_{i} \in\{0,1\}$ asymptotically converge.

Notice that, unlike Theorem 17, stubborn agents in the model (33) need not have identical opinions. In the case where $\lambda_{i}=1$ for any $i$, Theorem $18 \mathrm{im}$ plies Theorem 13 (for the Euclidean norm). In [155], the result of Theorem 18 has been extended to "anchored" HK systems, where opinions of each agent consists of a "mobile" part and static part; such systems appear to be equivalent, in some sense, to a special case of the heterogeneous model (20).

\subsubsection{Asymmetric interactions}

An important property of the HK model (11), (18), dramatically simplifying its analysis, is the symmetry of interactions. The influence graph $\mathcal{G}(x(k))$ is undirected, that is, at each step $k$ every two agents $i$ and $j$ either mutually influence each other or are independent. The modifications of the HK models with asymmetric interactions are much more complicated, and many of their properties observed in experiments are still waiting for mathematical investigation.

The simplest asymmetric bounded confidence model, proposed in [124], deals with scalar opinions $x_{i} \in \mathbb{R}$ and asymmetric confidence intervals, that is, the set of trusted individuals is defined as follows

$$
I_{i}(x)=\left\{j:-d_{l} \leq x_{j}-x_{i} \leq d_{r}\right\}, \quad d_{l}, d_{r}>0 .
$$

Obviously, in the case where $\varepsilon_{l} \neq \varepsilon_{r}$ the graph $\mathcal{G}(x(k))$ can be directed (which makes it impossible to apply Lemma 1, ensuring convergence). Also, the order of opinions in general is not preserved [133, 146]. Nevertheless, modification of the proof discussed in Subsect. 4.1 allows to show that the model (34) terminates in finite time; moreover, this holds for the more general class of asymmetric models with heterogeneous confidence intervals as follows

$$
I_{i}(x)=\left\{j:-d+\eta_{i} \leq x_{j}-x_{i} \leq d\right\}, \quad d, \eta_{i}>0 .
$$

Theorem 19. [133, 146] Assume that $\eta_{i} \geq 0 \forall i$ and $\eta=\max _{i} \eta_{i}<d$. Then the asymmetric $H K$ model (11), (35) terminates in finite time. 
As has been shown in [146], the termination time can be estimated as $O\left(n^{3}\right)+O\left(n^{2}\right) \ln (1-\zeta)$, where $\zeta=$ $\eta / d<1$, in particular, if $\zeta<1-\exp (-O(n))$ then the convergence time of the model is $O\left(n^{3}\right)$ like in the symmetric HK model $\left(\eta_{i}=0\right)$.

Obviously, Theorem 19 remains valid for the confidence intervals $\left(x_{i}-\varepsilon, x_{i}+\varepsilon-\eta_{i}\right)$ (which can be proved by changing the signs of opinions $x_{i} \mapsto-x_{i}$ ). However, allowing both left and right endpoints of the confidence intervals to be heterogeneous

$$
I_{i}(x)=\left\{j:-d_{l}^{i} \leq x_{j}-x_{i} \leq d_{r}^{i}\right\}, \quad d_{l}^{i}, d_{r}^{i}>0,
$$

one arrives at a very complicated system, still waiting for thorough analysis. Most typically, the confidence intervals are symmetric $d_{l}^{i}=d_{r}^{i}=d^{i}$ or, dealing with multidimensional opinions $x_{i} \in \mathbb{R}^{m}$, one ha: 10

$$
I_{i}(x)=\left\{j:\left\|x_{j}-x_{i}\right\| \leq d^{i}\right\} .
$$

Whereas the model (11), (37) has been proposed simultaneously with its homogeneous counterpart $\left(d^{i}=d\right)$ 124], its behavior in general "remains a mystery" [155]. Unlike the special case of the homogeneous model with stubborn agents $\left(d^{i} \in\{d, 0\} \forall i\right)$ discussed in the previous subsection, in general the convergence has been proved only for special solutions [135, 158], although simulations show that the convergence is a generic property of the heterogeneous HK model [158, 159] and its modifications [144, 157, 160]. An interesting phenomenon reported in [159] is emergence of consensus in the homogeneous HK model after injecting a very small proportion of agents with different confidence bound. Sufficient conditions for consensus in some heterogeneous HK models have been proposed in [161, 162].

\subsubsection{Other extensions}

The idea of bounded confidence, allowing to explain the phenomenon of persistent disagreement between opinions, has inspired numerous novel models of opinion formation. Most of them have been studied numerically and their mathematical properties have not been fully understood. For this reasons, the relevant works are only briefly mentioned.

Obviously, in reality social actors do not know the exact values of the others' opinions, which gives rise

\footnotetext{
${ }^{10}$ Along with bounded confidence model, a "bounded influence" model can be considered 158], replacing (37) by $I_{i}(x)=\left\{j:\left\|x_{j}-x_{i}\right\| \leq d^{j}\right\}$; the two models are equivalent in the homogeneous case, being quite different when $d^{i} \neq d^{j}$.
}

to the problem of robustness against various disturbances. Numerical simulations show high sensitivity of the HK dynamics to inaccuracies in the floating point arithmetic [82]. This is consonant with the recent analytic result [163], showing that small additive noises destroy clusters in the HK model and lead to "quasi-consensus". Similar effect is reported in [131] for stochastic differential equations, extending the continuous-time model (30). The model's ability to generate disagreement is however regained, allowing some non-local random interactions between the agents (an agent's opinion is not confined to the confidence interval) 80, 164 166]. Some extensions of the "truth-seeking" model (32), allowing random noises, have been proposed in [160, 167, 168].

Bounded confidence models appear to be related with community detection algorithms in graphs [169], Bayesian algorithms for distributed decision making [170] and algorithms of data clustering [171]. Bounded confidence models have been proposed for dynamics of "uncertain" opinions (standing for intervals of possible values) [172] and "linguistic" opinions representing words of a formal language [54, 173].

The HK model belongs to a family of so-called influence systems, introduced by B. Chazelle [174176] and generalizing a number of multi-agent algorithms arising in social and natural science. An influence system corresponds to the distributed protocol of iterative averaging, similar to the FrenchDeGroot and Abelson's models and their nonlinear counterparts [92, 107, 112, 113], over a state dependent graph. The existence of an arc in such a graph (that is, interaction between a pair of agents) is determined by some system of algebraic inequalities (strict or non-strict) with rational coefficients. The fundamental property of influence systems with bidirectional graphs is their asymptotic convergence (which has been shown for homogeneous HK model), whereas influence systems with directed graphs can exhibit very complex dynamics, being e.g. chaotic or Turing-complete (able to simulate any Turing machine) [175]. However, these "irregular" behaviors appear to be non-robust against small random perturbations, making almost all the trajectories of an influence system asymptotically periodic [175].

It should be noticed that introduction of the distant-based influence weights is not the only way to describe effects of homophily and biased assimilation in social groups, as illustrated by the recent work [120] that advocates a novel nonlinear extension 
of the DeGroot model to explain opinion polarization.

\section{Randomized Gossip-based Models}

The models considered in the previous sections adopt an implicit assumption of synchronous interactions among the agents. The agents simultaneously display their opinions to each other and simultaneously update them. Evidently, even for smallgroup discussions this assumption is unrealistic; as noticed in [84], "interpersonal influences do not occur in the simultaneous way... and there are more or less complex sequences of interpersonal influences in the group". One approach to portray these asynchronous and interactions among social actors is known as gossiping, assuming that agents interact not simultaneously but in in pairs. At any step, twd 11 agents interact (e.g. meet each other at some public place or communicate via phone/e-mail), after which one or both of their opinions can be changed.

Interest to gossip protocols has been stirred up by the following gossiping (or "telephone") problem in graph theory [178 180]. Suppose that each of $n$ people knows an item of scandal, which is not known to any of the others. They communicate by telephone, and whenever two individuals make a call, they pass on to each other, as much scandal as they know at that time. How many calls are needed before all the individuals know all the scandal? A more general problem with unidirectional communication has been addressed in [181, 182]. In the case of bidirectional information exchange (requiring undirected communication topology) and $n \geq 4$ the worst-case number of calls is $2 n-4$, whereas unidirectional communication over strongly connected directed graph requires, in general, $2 n-2$ calls 181, 182]. A survey of results on gossiping and a similar broadcasting problem (an item of information, known by one agent, has to be transmitted to all other agents) can be found in [183.

The pairwise gossiping interactions between the agents need not be random; consensus and other problems of multi-agent control can be solved e.g. by using periodic gossiping [184] and other distributed algorithms with deterministic asynchronous events [185, 186]. In this tutorial, we focus on randomized gossip-based models of opinion formation,

\footnotetext{
${ }^{11}$ Some results, discussed in this section, can be extended to the case of synchronous gossiping [177] where several dyadic interactions occur during each interaction session. For simplicity, we confine ourselves to the case of asynchronous interactions.
}

where random choice of the agents interacting at each step mimics spontaneity of real social interactions.

In this section, we suppose that the reader is familiar with the basic concepts of probability theory (probability spaces, random variables and their distributions, expectation and moments, convergence in probability and almost surely etc.) [187]. Henceforth $\mathbb{P}(A)$ denotes the probability of an event $A$ and $\mathbb{E} f$ stands for expectation of a random variable $f$.

\subsection{Gossip-based consensus}

In spite of relatively slow convergence, gossip-based consensus algorithms have attracted a lot of attention, being simple and very parsimonious in use of communication resources. The simplest linear gossiping algorithms can be considered as special cases of the French-DeGroot model (3) with random i.i.d 12 stochastic matrices $W(k)$. We first discuss some properties of such a randomized dynamics.

Definition 1. In the system (3), opinions are said to synchronize in probability, almost surely or in the $p$-th moment $(p>0)$ if for any $i, j=1, \ldots, n$ and any (deterministic) initial condition the sequence $x_{i}(k)-$ $x_{j}(k)$ converges in the corresponding sense, i.e.

$$
\begin{gathered}
\mathbb{P}\left(\left|x_{i}(k)-x_{j}(k)\right| \geq \varepsilon\right) \underset{k \rightarrow \infty}{\longrightarrow} 0 \forall \varepsilon>0 \quad \text { (in probability) } \\
\mathbb{P}\left(\lim _{k \rightarrow \infty}\left|x_{i}(k)-x_{j}(k)\right|=0\right)=1 \quad \text { (almost surely) } \\
\mathbb{E}\left|x_{i}(k)-x_{j}(k)\right|^{p} \underset{k \rightarrow \infty}{\longrightarrow} 0 \quad \text { (in the } p \text {-th moment) }
\end{gathered}
$$

The following fundamental result [177, 188, 189] (see also special cases in [190, 191]) extends Proposition 3 to the case of randomized French-DeGroot model, reducing it to a deterministic model.

Lemma 20. Consider the system (31) with i.i.d. stochastic matrices $W(k)$ and denote $\bar{W}=\mathbb{E} W(0)$. Then the following statements are equivalent

a) the deterministic French-DeGroot model

$$
x(k+1)=\bar{W} x(k), \quad k \geq 0
$$

reaches consensus, i.e. the stochastic matrix $\bar{W}$ is fully regular (or stochastic indecomposable aperiodic, SIA) [1];

b) the opinions reach consensus almost surely

$$
\mathbb{P}\left(\exists c \in \mathbb{R}: \lim _{k \rightarrow \infty} x(k)=c \mathbb{1}_{n}\right)=1 \quad \forall x(0) ;
$$

\footnotetext{
${ }^{12}$ Independent and identically distributed
} 
c) the opinions synchronize almost surely;

d) the opinions synchronize in probability;

e) the opinions synchronize in the p-th moment for some $p \in[1, \infty)$.

Furthermore, if (a)-(e) hold and $W(k)$ have a common nonnegative left eigenvector $w^{\top}$, such that $w^{\top} \mathbb{1}_{n}=1$, then in (39) one has $c=w^{\top} x(0)$.

The equivalence between (a) and (c)-(e) has been proved in [189]. Obviously, (b) implies (c). The last statement of Lemma 20 and the implication $(\mathrm{a}) \Longrightarrow(\mathrm{b})$ has been established in [188] under the additional assumption that $W(k)$ have strictly positive diagonal; the latter assumption can also be discarded [177] (formally, [177] establishes the implication (a) $\Longrightarrow$ (b) only for the case of irreducible $\bar{W}$, but the reducible case can be considered similarly). Consensus criteria, similar in spirit to Lemma 20, have been also established for non-stationary randomized models (where the i.i.d. assumption fails) [192, 193].

Remark 21. Under the assumptions of Lemma 20, $W(k)$ is independent of $x(k)$ (which depends only on $W(1), \ldots, W(k-1))$, which implies that the vectors $\mathbb{E} x(k)$ obey the deterministic model (38)

$$
\mathbb{E} x(k+1)=\mathbb{E} W(k) \mathbb{E} x(k)=\bar{W} \mathbb{E} x(k) \quad \forall k,
$$

in particular, the opinions' expectations $\mathbb{E} x_{i}(k)$ converge to consensus if (a)-(e) hold.

Consider now the following asynchronous gossip algorithm [177]. Consider a group of $n$ agents with opinions $x_{1}(k), \ldots, x_{n}(k) \in \mathbb{R}$ and a stochastic matrix $P=\left(p_{i j}\right)$ with zero diagonal $p_{i i}=0$. At each step $k$ of the opinion iteration, one agent $i=i(k)$ is randomly activated; we assume that the sequence $i(k)$ is i.i.d. and uniformly distributed in $\{1, \ldots, n\}$. With probability $p_{i j}$, the active agent $i$ interacts with agent $j$ and updates its opinion as follows

$$
x_{i}(k+1)=\left(1-\gamma_{i}\right) x_{i}(k)+\gamma_{i} x_{j}(k),
$$

where $\gamma_{i} \in(0,1)$ is a constant, describing the "trust" of individual $i$ in his/her neighbors. The opinions of the other agents (including $j$ ) remain unchanged

$$
x_{l}(k+1)=x_{l}(k) \quad \forall l \neq i(k) .
$$

It can be easily shown that the algorithm (40), (41) is a special case of (3), where $W(k)$ are i.i.d. stochastic matrices, attaining one of the values $W^{i j}=$
$I_{n}+\gamma_{i}\left(e_{j}-e_{i}\right) e_{i}^{\top}$ with the probability and $\mathbb{P}(W(k)=$ $W^{i j}$ ) $=n^{-1} p_{i j}$ (here $e_{i} \in \mathbb{R}^{n}$ is the column vector with all components equal to 0 except for the $i$ th component, which is equal to 1$)$. Introducing the diagonal matrix $\Gamma=\operatorname{diag}\left(\gamma_{1}, \ldots, \gamma_{n}\right)$, we have

$$
\bar{W}=\mathbb{E} W(1)=I_{n}-n^{-1} \Gamma+n^{-1} \Gamma P .
$$

Obviously, the diagonal entries of $\bar{W}$ are positive and when $i \neq j$, one has $\bar{w}_{i j}>0 \Leftrightarrow p_{i j}>0$. Hence, the system (38) reaches consensus if and only if the graph $\mathcal{G}(P)$ has a directed spanning tree (see [1]). Lemma 20 yields in the following consensus criterion.

Corollary 22. For any gains $\gamma_{1}, \ldots, \gamma_{n}$ and stochastic matrix $P$, such that the graph $\mathcal{G}(P)$ has a directed spanning tree, the protocol (40), (411) provides consensus of opinions with probability 1 (39).

Along with unidirectional gossip algorithm, one may consider bidirectional protocol where both agents $i, j$ update their opinions. The simplest algorithm of this type has been examined in 194]

$$
\begin{gathered}
x_{i}(k+1)=x_{j}(k+1)=\frac{x_{i}(k)+x_{j}(k)}{2} \\
x_{l}(k+1)=x_{l}(k) \quad \forall l \neq i, j .
\end{gathered}
$$

It can be shown [194] that in this situation one has

$$
\bar{W}=I-\frac{1}{2 n} D+\frac{1}{2 n}\left(P+P^{\top}\right),
$$

where $D$ is the diagonal matrix with entries $d_{i i}=$ $1+\sum_{j} p_{j i} \leq 1+n$. Consensus in (38) is established if and only if the undirected graph, corresponding to $P+P^{\top}$, is connected.

A more advanced analysis of gossip-based consensus algorithms and overview of their applications are available in [177, 184, 194 197] and references therein.

\subsection{Gossiping with stubborn agents}

As has been in discussed in Part I [1], the presence of several stubborn agents in the static FrenchDeGroot model (1) yields in more interesting dynamics than conventional consensus algorithms exhibit: opinions do not reach consensus and typically split into several clusters (this holds when the matrix $W$ is regular, i.e. the limit $\lim _{k \rightarrow \infty} W^{k}$ exists). A natural question arises whether its gossip-based counterpart exhibits a similar behavior. The answer to this question appears to be negative: as has been shown 
in [198], in presence of stubborn agents the gossip algorithms fail to provide almost sure convergence of the opinions, which keep on fluctuating in an ergodic fashion. At the same time, the distribution of the opinion vector $x(k)$ converges to some probability measure. In this subsection, we discuss similar result 13 , concerning the asynchronous gossip-based version of the Friedkin-Johnsen (FJ) model [199 203].

\subsubsection{Example: fluctuation between two stubborn leaders and Bernoulli convolution series}

We start with an example from [198], demonstrating that in presence of stubborn agents the gossip algorithm does not provide convergence of the opinions. Consider two stubborn individuals with fixed opinions $x_{1} \equiv 0$ and $x_{2} \equiv 1$ and "regular" agent that can interact with both of them. At each step $k$, the regular agent chooses one of the stubborn neighbors $i=i(k) \in\{1,2\}$ with probability $1 / 2$ and shifts its own opinion towards the opinion of this neighbor

$$
x_{3}(k+1)=(1-\gamma) x_{3}(k)+\gamma x_{i(k)}, \quad \gamma \in(0,1) .
$$

Since $x_{3}(k)=(1-\gamma)^{k} x_{3}(0)+\gamma \sum_{s=0}^{k-1}(1-\gamma)^{s} x_{i(k-s)}$, it can be shown that the distribution of $x_{3}$ converges 14 as $k \rightarrow \infty$ to the distribution of the random variable

$$
\bar{x}_{3}=\frac{\gamma}{1-\gamma} \bar{\xi}, \quad \bar{\xi}=\sum_{s \geq 1}(1-\gamma)^{s} \xi_{s},
$$

where $\xi_{j}$ are i.i.d. Bernoulli random variables with probability $1 / 2$. The random variable $\bar{\xi}$ in the righthand side is referred to as the Bernoulli convolution 204]. For $\gamma=1 / 2, \bar{\xi}$ is uniformly distributed on $[0,1]$. For almost all $\gamma \in(0,1 / 2)$ its distribution on $[0,1]$ is absolutely continuous (has a density), whereas for $\gamma>1 / 2$ it is supported on the Cantor set [204].

This example demonstrates, in particular, that consensus in Lemma 20 cannot be replaced by convergence of the opinions: such a convergence in the

\footnotetext{
${ }^{13}$ Technically the model in [198] differs from the models considered in this subsection, e.g. it deals with dynamics on a continuous time scale, where interactions between pairs of connected agents are activated by clocks, each ticking at the times of an independent Poisson process of certain rate. Some results from [198], concerned with the characteristics of the stationary distribution, still have not been extended to the FJ model.

${ }^{14}$ Henceforth, by convergence of distributions we mean the standard weak convergence: a sequence of probability measures $\mathbb{P}_{k}$ on the same $\sigma$-algebra converges to a measure $\mathbb{P}$, if $\mathbb{E}_{\mathbb{P}_{k}} f \rightarrow$ $\mathbb{E}_{\mathbb{P}} f$ as $k \rightarrow \infty$ for any bounded random variable $f$.
}

deterministic model (38), in general, does not imply the convergence of the model (3) with random $W(k)$. At the same time, Remark 20 implies that $e x$ pected values of the opinions converge. For the special gossip-based model considered below, the convergence of time averages can also be proved.

\subsubsection{The asynchronous gossip-based FJ model}

Recall that the FJ model [84] is characterized by two matrices $\Lambda, W$, where $\Lambda$ is a diagonal matrix of agents' susceptibilities to social influence, $0 \leq \Lambda \leq$ $I_{n}$, and $W$ is a stochastic matrix of influence weights. The opinion vector $x(k) \in \mathbb{R}^{n}$ evolves as follows

$$
x(k+1)=\Lambda W x(k)+(I-\Lambda) u, \quad u=x(0) .
$$

The relations between the FJ model and the FrenchDeGroot model has been discussed in Part I [1], as well as the graphical conditions for the model's stability and convergent. In this subsection, we assume that the model (43) is a stable 15 as a system with static input $u$, that is, $\Lambda W$ is a Schur stable matrix $\rho(\Lambda W)<1$, and hence the opinions in (43) converge

$$
x(k) \underset{k \rightarrow \infty}{\longrightarrow} \bar{x}=(I-\Lambda W)^{-1}(I-\Lambda) u .
$$

Numerous experiments with small and mediumsize group [84, 85, 205, 206] have confirmed the predictive power of the FJ model, in particular, (44) gives a good approximation for the real distribution of final opinions. This means that the asynchronous gossip-based counterpart of the FJ model should also provide (in some sense) the correspondence (44) between the "prejudice" vector $u=x(0)$ and the outcome of the opinion formation process. We consider one such gossip-based model, proposed in 203] and generalizing the protocols from 199 201].

Let $\mathcal{G}[W]=(\mathcal{V}, \mathcal{E})$ stand for the interaction graph of the network and consider two matrices

$$
\Gamma^{1}=\Lambda W, \quad \Gamma^{2}=(I-\Lambda) W .
$$

Consider the following asynchronous gossip-based algorithm, similar in structure to (40), (41). At each step $k$, an arc is uniformly sampled in the set $\mathcal{E}$. If

\footnotetext{
${ }^{15}$ As shown in [1, 203], unstable FJ model (with $\rho(\Lambda W)=$ 1) contains a subgroup of agents obeying the usual FrenchDeGroot model and independent of the remaining agents. Such situation is impossible, e.g. when $\Lambda \neq I$ and $W$ is irreducible.
} 
this arc is $(i, j)$, then agent $i$ communicates to agent $j$ and updates its opinion as follows

$$
x_{i}(k+1)=\left(1-\gamma_{i j}^{1}-\gamma_{i j}^{2}\right) x_{i}(k)+\gamma_{i j}^{1} x_{j}(k)+\gamma_{i j}^{2} u_{i} .
$$

Hence during each interaction the agent's opinion is averaged with its own prejudice and the neighbors' opinion; note, however, that the weight of the prejudice depends on both $\lambda_{i i}$ and $w_{i j}$. The other opinions (including opinion of agent $j$ ) remain unchanged

$$
x_{l}(k+1)=x_{l}(k) \quad \forall l \neq i .
$$

The following theorem shows that the gossiping opinion formation process (46), (47) inherits the properties of the FJ model on average. Let $\bar{x}(k)$ stand for the Cesáro (or Polyak) average

$$
\bar{x}(k):=\frac{1}{k+1} \sum_{l=0}^{k} x(l) .
$$

Theorem 23. [203] Let $\rho(\Lambda W)<1$ and $\Gamma^{1}, \Gamma^{2}$ be matrices from (45). Then, there is the following correspondence between the gossip-based model (46), (47) and the deterministic model (43):

1. the steady expected value $\lim _{k \rightarrow \infty} \mathbb{E} x(k)=\bar{x}$ coincides with (44) for any $x(0)$;

2 . the random process $x(k)$ is almost sure ergodic

$$
\mathbb{P}\left(\lim _{k \rightarrow \infty} \bar{x}(k)=\bar{x}\right)=1 ;
$$

3. for any $p>0$, the process $x(k)$ is $L^{p}$-ergodic

$$
\mathbb{E}|\bar{x}(k)-\bar{x}|^{p} \underset{k \rightarrow \infty}{\longrightarrow} 0 .
$$

Additionally, the process $x(k)$ converges in distribution to some random vector $x_{\infty}$ with $\mathbb{E} x_{\infty}=\bar{x}$, whose distribution is determined by $\Lambda, W$.

As noticed in [203], the statements of Theorem 23 remain valid for any matrix $\Gamma^{2}$ such that $0 \leq \gamma_{i j}^{2} \leq$ $1-\gamma_{i j}^{1}, \sum_{j=1}^{n} \gamma_{i j}^{2}=1-\lambda_{i i}$ and $\gamma_{i j}^{2}=0$ as $(i, j) \notin \mathcal{E}$. In [203], multidimensional gossip-based models have been also considered, where opinions represent individuals' positions on several interrelated topics (belief systems [44]). In [199], the estimate for the convergence rate (and variance) of $\bar{x}(k)$ has been obtained

$$
\mathbb{E}|\bar{x}(k)-\bar{x}|^{2} \leq \frac{\chi}{k+1},
$$

where $\chi$ depends on $u$ and the spectral radius $\rho(\Lambda W)$ (in fact, $\chi \rightarrow \infty$ as $\rho \rightarrow 1$ ). The proof of Theorem 23 is based on the elegant results of [200], dealing with the properties of randomized affine system

$$
\xi(k+1)=M(k) \xi(k)+u(k),
$$

with special i.i.d. sequences of the matrices $M(k)$ (non-stochastic) and vectors $u(k)$ with finite expectations. The key property of the system (49), needed for the existence of a stationary distribution and ergodicity of its solutions is the decomposability

$$
\mathbb{E} M(k)=\alpha I+(1-\alpha) P,
$$

where $\alpha \in(0,1)$ and $\operatorname{det}(I-P) \neq 0$.

Similar to the example with three agents from previous subsection, opinion vectors $x(k)$ usually do not converge and keep on fluctuating, as demonstrated by the following example [203]. Consider $n=4$ agents with the initial opinions $u=x(0)=(25,25,75,85)^{\top}$ and the matrix $W$, observed in a real social group [84]

$$
W=\left[\begin{array}{cccc}
0.220 & 0.120 & 0.360 & 0.300 \\
0.147 & 0.215 & 0.344 & 0.294 \\
0 & 0 & 1 & 0 \\
0.090 & 0.178 & 0.446 & 0.286
\end{array}\right]
$$

The matrix $\Lambda$ is defined by the "coupling condition" $\Lambda=I-\operatorname{diag} W$. The final opinion vector (44) of the FJ model is $\bar{x} \approx(60,60,75,75)^{\top}$, i.e. agents 1 and 2 form their own cluster, whereas stubborn agent 3 attracts the opinion of agent 4. Fig. 4 illustrates the trajectories of the deterministic and randomized FJ models, as well as the Cesáro averages $\bar{x}(k)$. As shown in Fig. 4k, the opinions fluctuate (except for the opinion of stubborn agent 3 ).

A similar behavior is demonstrated by the randomized algorithms for PageRank computation [207 209], whose relation to the asynchronous gossip-based FJ model has been disclosed in [200, 201].

\subsection{The Deffuant-Weisbuch model}

Along with the HK model discussed Section 4, the Deffuant-Weisbuch (DW) model is among the most illustrious models of opinion formation. Being a gossip-based counterpart of the HK model, the DW model in fact was proposed in [123] independent of Krause's work [122], employing the same principle of bounded confidence. In spite of many numerical results and experimental observations, dealing with the behavior of the DW model and its modifications 


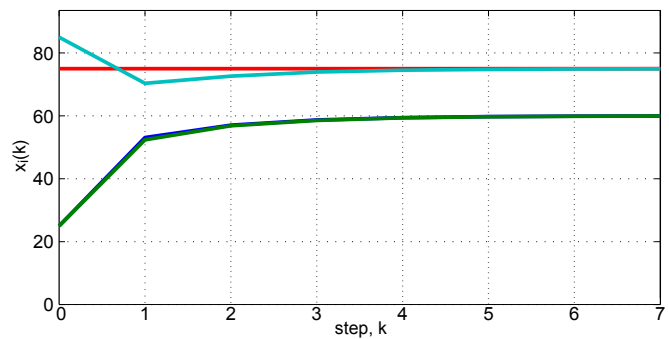

(a) Deterministic model

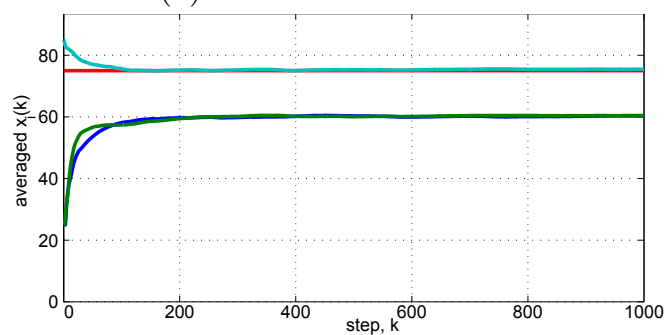

(b) Averaged random opinions $\bar{x}(k)$

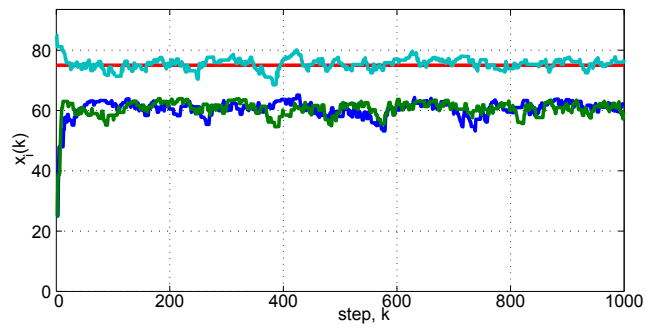

(c) Random opinions $x(k)$

Figure 4: Deterministic FJ model (43) vs. the asynchronous gossip-based model (46), (47).

over complex networks [123, 210 215], the compound of randomness and nonlinear dynamics makes these models very hard for mathematical investigation, and analytic results explaining their behavior are limited.

The original DW model has a structure similar to the symmetric gossiping protocol (42), however, the interaction graph is coincident with the graph $\mathcal{G}(x(k))$ introduced for the HK model in Section 4, being state-dependent and determined by the distances between the opinions. Similar to the HK model, the DW model [123] deals with a group of $n$ agents, each having the confidence bound $d>0$ (referred also to as the agent's "threshold" [123] or "uncertainty" 210]). The opinions $x_{i} \in \mathbb{R}$ are updated in accordance with the gossiping procedure as follows.

At each step, a pair of agents $(i, j)$ is chosen randomly. These two agents interact if and only if their opinions are close. Denoting the indicator function of

\footnotetext{
${ }^{16}$ For simplicity, we confine ourselves to scalar DW model, although vector opinions have been also introduced in [123].
}

an event $\Omega$ with $\mathbb{I}(\Omega)$, the DW model is as follows

$$
\begin{gathered}
x_{i}(k+1)=x_{i}(k)+\mu\left(x_{j}(k)-x_{i}(k)\right) \mathbb{I}_{k}, \\
x_{j}(k+1)=x_{j}(k)+\mu\left(x_{i}(k)-x_{j}(k)\right) \mathbb{I}_{k}, \\
x_{l}(k+1)=x_{l}(k) \quad \forall l \neq i, j, \\
\mathbb{I}(k)=\mathbb{I}\left(\left|x_{j}(k)-x_{i}(k)\right| \leq d\right) .
\end{gathered}
$$

The constant $\mu \in(0,1)$ is called convergence parameter [123] and stands for the attraction between the opinions. Similarly to the models from the previous subsections, (50) can be considered as the FrenchDeGroot model with state-dependent random matrices $W(k)=W(x(k))$. Such matrices are no longer i.i.d. since $W(k)$ depends on $W(k-1)$, however, $x(k)$ remains a Markov process, and for any its realization the matrices $W(k)$, as can be easily shown, satisfy the conditions of Lemma 1, entailing in the following.

Lemma 24. The opinions in the model (50) converge almost surely, that is

$$
\mathbb{P}\left(\exists \bar{x}_{i}=\lim _{k \rightarrow \infty} x_{i}(k)\right)=1 \quad \forall i .
$$

Furthermore, for any $i, j$ one almost surely has either $\left|\bar{x}_{i}-\bar{x}_{j}\right| \geq d$ or $\bar{x}_{i}=\bar{x}_{j}$.

The first statement of Lemma 24 is immediate from Lemma 1. To prove the second statement, consider a pair of indices $\left(i_{0}, j_{0}\right)$. Due to the Borel-Cantelli lemma [187], $(i, j)=\left(i_{0}, j_{0}\right)$ infinitely often with probability 1 . Hence either agents $\left(i_{0}, j_{0}\right)$ are infinitely connected in the time-varying graph $\mathcal{G}(W(k))$ (and then $\bar{x}_{i_{0}}=\bar{x}_{j_{0}}$ ) or the sequence $k_{s} \rightarrow \infty$ exists such that $\left|x_{i_{0}}\left(k_{s}\right)-x_{j_{0}}\left(k_{s}\right)\right|>d$, i.e. $\left|\bar{x}_{i_{0}}-\bar{x}_{j_{0}}\right| \geq d$.

An important and non-trivial result from [216], based on techniques from [193], shows that Lemma24] retains its validity for the extensions of (50) that allow asymmetric interactions among the agents. The simplest of such models is similar to (50) yet assumes that only agent $i$ updates its opinion, and the other opinions remain constant

$$
\begin{gathered}
x_{i}(k+1)=x_{i}(k)+\mu\left(x_{j}(k)-x_{i}(k)\right) \mathbb{I}_{k}, \\
x_{l}(k+1)=x_{l}(k) \quad \forall l \neq i \\
\mathbb{I}_{k}=\mathbb{I}\left(\left|x_{j}(k)-x_{i}(k)\right| \leq d\right) .
\end{gathered}
$$

More complicated are "multi-choice" [216] extensions of (51), allowing an "active" agent $i$ to interact with several neighbors. Lyapunov techniques allow to obtain some explicit estimates for the convergence rate of (50) and its asymmetric counterparts [217]. 
Similar to the case of HK model, heterogeneous confidence bounds $d_{i}$ in the DW model lead to serious complications. The heterogeneous counterpart of (50), studied in [159, 218], is as follows

$$
\begin{gathered}
x_{i}(k+1)=x_{i}(k)+\mu\left(x_{j}(k)-x_{i}(k)\right) \mathbb{I}_{k}^{i}, \\
x_{j}(k+1)=x_{j}(k)+\mu\left(x_{i}(k)-x_{j}(k)\right) \mathbb{I}_{k}^{j}, \\
x_{l}(k+1)=x_{l}(k) \quad \forall l \neq i, j, \\
\mathbb{I}_{k}^{i}=\mathbb{I}\left(\left|x_{j}(k)-x_{i}(k)\right| \leq d_{i}\right), \\
\mathbb{I}_{k}^{j}=\mathbb{I}\left(\left|x_{j}(k)-x_{i}(k)\right| \leq d_{j}\right) .
\end{gathered}
$$

As reported in [218], in presence of "extremists" with very narrow confidence intervals, the model (52) exhibits ergodic fluctuations of the opinions, similar to the behavior of asynchronous gossip-based models with stubborn agents [198] and the FJ model (46).

Even more sophisticated are extensions of the DW models, involving nonlinear couplings among interacting agents (e.g. the "relative agreement" models [210, 214, 215, 219]), dynamic or random confidence bounds [211, 220, 221], noises [222, 223] and "long-range" interactions (agents can assimilate opinions beyond their confidence intervals) [216, 223]. These models are beyond the scope of this tutorial, as well as the gossip-based counterparts of the "truthseeking" model (32), introduced in [224, 225].

\section{Disagreement via Negative Influence}

The models discussed in the previous section extend the basic French-DeGroot and Abelson models, inheriting however the key idea of iterative averaging. Even though agreement is not always possible (due to the effects of stubborness, homophily etc.), the agents cooperate in order to reach it, always changing their opinions towards each other. In many systems, arising in economics, natural sciences and robotics such positive (attractive) couplings among the agents coexist with negative (repulsive) couplings, leading to the agents' distancing. These negative ties among the agents can correspond to their competition; multi-agent networks where agents can both cooperate and compete are sometimes called "coopetitive" 226, 227]. Repulsive interactions lie in the heart of many biological system [228, 229] and are vital for collision avoidance in swarms and robotic formations [230 232]. Unlike the well-developed theory of cooperative networks, the studies on "coopetitive" networks are taking their first steps.
As a possible reason for disagreement of opinions, Abelson [72] mentions the boomerang effect [233, 234], being an unintended consequence of the persuasion process. An attempt to persuade a person sometimes shifts his/her opinion away from the persuader's opinion. The boomerang effects can be partly explained by personal insults 235] occuring during the discussion or the discussants' reactanct17 [236]. The theories of balance [237] and cognitive dissonance 238] explain the presence of negative influences by coevolution of the opinions and personal relations between individuals. An agreement with negatively evaluated person creates a psychological tension, and individuals can resolve such tensions by "moving their opinions away from a disliked source" [239]. Models proposed in 240 242] postulate that negative ties between individuals arise due to large discrepances of their opinions. Unlike the bounded confidence models, an individual does not reject too dissimilar opinions but rather shifts his/her opinion away from them. These and many other models of opinion formation under negative influence, offered in the literature and still awaiting rigorous analysis [119, 243245], illustrate that antagonism among the agents may lead to the community cleavage.

The ubiquity of such negative ties has not secured by experimental evidence, e.g. the recent experiments reported in [239] does not support the aforementioned hypotheses, explaining negative influence to dissimilarities and disliking. Nevertheless, from the authors' point of view, models of opinion formation with antagonistic interactions deserve attention since they exhibit non-trivial behaviors and can lead to the development of novel mathematical theories. In this section, we consider the model proposed by Altafini [246, 247] and its extensions. The idea of opinion polarization via structural balance has triggered the extensive research on "bipartite" consensus, synchronization and flocking [248 250], extending the relevant results of multi-agent cooperative control to networks with antagonistic interactions.

\subsection{Balance theory}

The idea of Altafini's model has been inspired by the theory of structural balance, pioneered in [237,

\footnotetext{
${ }^{17}$ The reactance is an individual's ability to resist the persuasion, being heavily pressed to accept some attitude. This resistance may lead, under some circumstances, to adoption of an attitude, which is opposite to the persuader's one.
} 
251, 252] and postulating the mutual dependence between the interpersonal relations and the opinion formation: "an attitude towards an event can alter the attitude towards the person who caused the event, and, if the attitudes towards a person and an event are similar, the event is easily ascribed to the person" [237]. Positive and negative evaluations of the individuals by each other are naturally represented by signed graphs.

Definition 2. A signed graph is a triple $\mathcal{G}=$ $(V, E, A)$, where $(V, E)$ is a graph and $A=\left(a_{i j}\right)_{i, j \in V}$ is matrix, whose entries may be negative, such that $a_{i j} \neq 0$ if and only if $(j, i) \in E$. An arbitrary matrix $A=\left(a_{i j}\right)_{i, j \in V}$ corresponds to the unique signed graph $\mathcal{G}[A]=(V, E[A], A)$, where $E[A]=\left\{(i, j): a_{j i} \neq 0\right\}$.

One can interpret positive arcs as relations of friendship or trust, negative ones standing for enmity or suspicion. We call arc $(i, j)$ of a signed graph positive (negative) if its "weight" $a_{i j}$ is positive (negative). Henceforth, unless stated otherwise, we assume that all signed graphs are sign-symmetric [247] in the sense that $a_{i j} a_{j i} \geq 0$ (in other words, the relations of friendship and enmity are symmetric). This condition is necessary for the structural balance and allows to simplify some constructions.

To illustrate the notion of structural balance, consider for the moment a complete signed graph. The structural balance in such a graph can be defined as balance in each triad (subgraph with three nodes), which is understood as follows [237, 252].

Definition 3. A complete sign-symmetric graph is structurally balanced if each triad with nodes $(i, j, k)$ is balanced in the sense that $a_{i j} a_{j k} a_{k i}>0$ (Fig 5).

A balanced triad (Fig. 5a,b) represent either a triple of "friends" (a) or an alliance of two "friends" against a common "enemy" (b). The imbalance in a triad (Fig. [5],d), violating the ancient proverb the friend of my enemy is my enemy, the enemy of my enemy is my friend [253], creates social tensions which social actors, according to balance theory, tend to resolve. Whereas structural balance is inherent to many real-world networks [11, 254, 255], classical balance theory does not explain how the system reaches the balanced state. Dynamics of weights $a_{i j}$ that lead to their structural balance are beyond the scope of this tutorial and can be found e.g. in 256 263.

It can be seen that the nodes of a structurally balanced sign-symmetric complete graph split into two

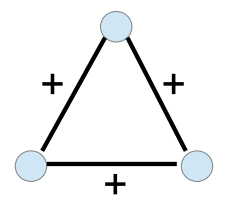

(a)

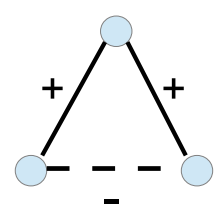

(c)

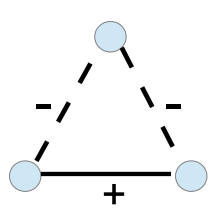

(b)

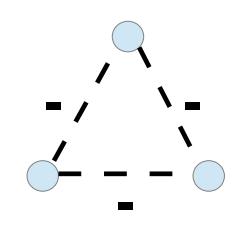

(d)
Figure 5: Balanced vs. imbalanced triads

factions, or "hostile camps" 264] as follows. Consider an arbitrary agent $i$ and let the set $V_{1}$ consist of $i$ and his/her friends $V_{1}=\{i\} \cup\left\{j \neq i: a_{i j}>0\right\}$, while the set $V_{2}$ consists of his/her enemies $V_{2}=$ $\left\{j \neq i: a_{i j}<0\right\}$. By definition of the structural balance, every two friends, as well as every two enemies, of agent $i$ are friends, i.e. $a_{j k}>0$ whenever $(j, k) \in\left(V_{1} \times V_{1}\right) \cup\left(V_{2} \times V_{2}\right)$ and $j \neq k$. Similarly, a friend of $i$ and an enemy of $i$ are always enemies: $a_{j k}<0$ when $(j, k) \in\left(V_{1} \times V_{2}\right) \cup\left(V_{2} \times V_{1}\right)$ and $j \neq k$. The converse statement is also valid: if the set of nodes $V$ can be decomposed into two disjoint sets $V_{1}, V_{2}$ with the aforementioned properties, then the graph is structurally balanced. Indeed, in each triad two nodes belong to the same "camp". The remaining node can either belong to the same camp or the opposite camp, which corresponds, respectively, to the triads of types (a) and (b) in Fig. 5. This result, established in [265] and called the "balance theorem", inspires the following general definition.

Definition 4. (Structural balance) A general signed graph is $G=(V, E, A)$ is structurally balanced if the set of its nodes can be divided into such disjoints subsets $V=V_{1} \cup V_{2}$ that for any pair $i, j \in V, i \neq j$,

$$
\begin{cases}a_{i j} \geq 0, & \text { if } \quad(i, j) \in\left(V_{1} \times V_{1}\right) \cup\left(V_{2} \times V_{2}\right), \\ a_{i j} \leq 0, & \text { if } \quad(i, j) \in\left(V_{1} \times V_{2}\right) \bigcup\left(V_{2} \times V_{1}\right) .\end{cases}
$$

Note that one of the "camps" $V_{i}$ may be empty (this holds in the case of a usual weighted graph, where the matrix $A$ is nonnegative). Unlike the case of a complete graph, balance in each triad is insufficient for structural balance in the graph. To formu- 
late a necessary and sufficient condition, we introduce two auxiliary concepts.

Definition 5. For a sign-symmetric graph $\mathcal{G}=$ $(V, E, A)$, consider an undirected graph $\hat{\mathcal{G}}=$ $\left(V, \hat{E}, A+A^{\top}\right)$ obtained from $\mathcal{G}$ by "mirroring" each directed arc 18 A semiwalk $v_{0}, \ldots, v_{n}$ in the graph $\mathcal{G}$ is a sequence of nodes, corresponding to a walk in $\hat{\mathcal{G}}$; if $v_{0}=v_{n}$, this semiwalk is called semicycle.

Definition 6. We call a walk $v_{0}, \ldots, v_{n}$ is a signed graph $\mathcal{G}$ positive (respectively, negative) if the product of all weights on the connecting arcs $a_{v_{0} v_{1}} a_{v_{1} v_{2}} \ldots a_{v_{n-1} v_{n}}$ is positive (negative). The sign of a semiwalk in a sign-symmetric graph $\mathcal{G}$ is its sign as a walk in the corresponding undirected graph $\hat{\mathcal{G}}$.

It can be shown (using e.g. induction on the walk's length) that a negative walk in a structurally balanced graph connects the nodes from different camps, whereas a positive walk starts and ends at the same camp. In particular, for structural balance it is necessary that the all cycles are positive (for cycles of length 2, this implies the sign symmetry of the graph). This is condition is also sufficient for strongly connected graphs [247], however, without strong connectivity, even graphs with $n=3$ nodes can be structurally imbalanced and have no cycles (see Fig. 6). By noticing that the undirected graph $\hat{\mathcal{G}}$, corresponding to a structurally balanced graph $\mathcal{G}$, is also structurally balanced, one shows that in fact all semicycles have to be positive, and this condition appears to be sufficient for structural balance [252].

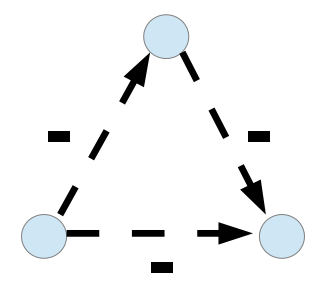

Figure 6: A graph without cycles, no structural balance

Lemma 25. 19 If a signed graph is structurally balanced, then all its cycles are positive (in particular, the graph is sign-symmetric). This condition is

\footnotetext{
${ }^{18}$ Since $a_{i j} a_{j i} \geq 0$ for $i \neq j,\left(A+A^{\top}\right)_{i j} \neq 0$ if and only if $\left|a_{i j}\right|+\left|a_{j i}\right| \neq 0$. In other words, $(i, j) \in \hat{E} \Leftrightarrow(j, i) \in \hat{E} \Leftrightarrow$ $(i, j) \in E \vee(j, i) \in E$, so the undirected graph $\hat{\mathcal{G}}$ contains all directed $\operatorname{arcs}(i, j)$ of $\mathcal{G}$ together with their "mirrors" $(j, i)$.

${ }^{19}$ The necessary and sufficient condition from this lemma is often used as a definition of structural balance [252].
}

also sufficient for structural balance when the graph is strongly connected. In general, for structural balance it is necessary and sufficient that the graph is sign-symmetric and all its semicycles are positive.

The concept of structural balance has been extended in various directions, e.g. the more general condition of weak balance allows clustering into more than two factions [266], where members of each factions are friends and the members of different factions are enemies. For undirected graphs, the weak balance is equivalent to the absence of cycles with exactly one negative arc 266]. A natural question, concerned with imbalanced signed graph, is how to measure the "level of imbalance" (or how close the graph is to balance). Different measures of imbalance have been proposed in [11, 254, 255, 267].

Following [247], we define the Laplacian matrix $L=L[A]$ of the signed graph $\mathcal{G}=(V, E, A)$ as follows

$$
L[A]=\left(l_{j k}\right), l_{j k}=\left\{\begin{array}{l}
-a_{j k}, j \neq k \\
\sum_{m=1}^{N}\left|a_{j m}\right|, j=k .
\end{array}\right.
$$

In the case where $A$ is nonnegative, (53) coincides with the conventional Laplacian matrix of a weighted graph [1]. As implied by the Gershgorin Disc Theorem, $L[A]$ has no eigenvalues in the closed left halfplane $\overline{\mathbb{C}}_{-}=\{\lambda \in \mathbb{C}: \operatorname{Re} \lambda \leq 0\}$ except for, possibly, $\lambda=0$. Unlike the unsigned case, in general $L[A]$ can have no zero eigenvalue, in which case the matrix $(-L[A])$ is Hurwitz.

The following elegant result [247] establishes a relation between the Laplacian and structural balance.

Lemma 26. For a strongly connected signed graph $\mathcal{G}=(V, E, A), \lambda=0$ is an eigenvalue of $L[A]$ if and only if the graph $\mathcal{G}$ is structurally balanced.

As will be shown, the sufficiency part in Lemma 26 in fact does not rely on the strong connectivity. The "gauge transformation" 247] introduced in the next subsection transforms the Laplacian of a structurally balanced graph $L[A]$ into the matrix $L\left[A^{|\cdot|}\right]$, where $A^{|\cdot|}=\left(\left|a_{i j}\right|\right)$. In other words, $L[A]$ is similar to the Laplacian of an (unsigned) weighted graph, which always has an eigenvalue at 0 .

\subsection{Altafini's model of opinion formation}

Balance theory suggests that a natural outcome of the opinion formation process should somehow reflect 
the partition of the network into two opposing factions [246]. In the models offered in [246, 247], structural balance of a signed network leads to polarization ("bipartite consensus") of the opinions: opinions in each faction reach consensus, and their consensus values are equal in modulus yet differ in sign.

The origina 20 Altafini model [247] is coincident with the Abelson model (8), however the matrix $A(t)$ need not be nonnegative, and the corresponding Laplacian is understood in the sense of (53)

$$
\begin{gathered}
\dot{x}_{i}(t)=\sum_{j=1}^{n}\left|a_{i j}(t)\right|\left(x_{j}(t) \operatorname{sgn} a_{i j}(t)-x_{i}(t)\right)= \\
=\sum_{j=1}^{n}\left(a_{i j}(t) x_{j}(t)-\left|a_{i j}(t)\right| x_{i}(t)\right) \forall i, \quad \stackrel{(55)}{\Longrightarrow} \\
\Longleftrightarrow \dot{x}(t)=-L[A(t)] x(t), \quad t \geq 0 .
\end{gathered}
$$

To ensure the existence and uniqueness of the solution for any $x(0)$, it suffices to assume that $A(\cdot)$ is locally $L_{1}$-function. Similar to the Abelson model, solutions of the Altafini model are globally bounded; it can be shown [264] that $M(t)=\max _{i}\left|x_{i}(t)\right|$ is a non-increasing function and hence $\left|x_{i}(t)\right| \leq M(0)$ for any $i$ and $t \geq 0$, entailing the following proposition.

Proposition 27. System (54) is Lyapunov stable.

As we will see, unlike the unsigned case the system (54) can, in general, be asymptotically stable.

\subsubsection{The case of a time-invariant signed graph}

We start with analysis of the static Altafini model $(A(t) \equiv A$ is a constant matrix). Similar to the static Abelson model, the opinions always converge.

Proposition 28. For any matrix $A$ and initial condition $x(0)$, there exist finite limits $\bar{x}_{i}=\lim _{t \rightarrow \infty} x_{i}(t)$.

Proposition 28 is immediate from Proposition 27. If $L[A]$ has an eigenvalue at 0 , the Jordan cells corresponding to it are trivial in view of the Lyapunov stability. Since all other eigenvalues of $(-L[A])$ are stable, this implies the existence of $\lim _{t \rightarrow \infty} e^{-L[A] t}$.

Proposition 28 can be also proved by using the lifting approach [270], showing that the Altafini model is equivalent to Abelson's model with $2 n$ agents $1, \ldots, n$

\footnotetext{
${ }^{20}$ In this tutorial, we confine ourselves to the linear Altafini model and do not consider its nonlinear counterparts 246, 247, 264, 268, 269], whose properties are in fact very similar.
}

and $1^{\prime}, \ldots, n^{\prime}$, where agent $i^{\prime}$ is the antipode of agent $i$ and has opinion $x_{i^{\prime}}=\left(-x_{i}\right)$. Some other results, concerned with the behavior of Altafini's model, can also be derived by using this lifting technique. Note that the arguments, used in [1] to prove the convergence of static Abelson models, cannot be extended to the signed case since $L[A]$ is no longer a $M$-matrix.

The Altafini model has the most interesting behavior when the graph $\mathcal{G}=(V, E, A)$ is structurally balanced. Consider the decomposition of $V$ into two "hostile camps" $V=V_{1} \cup V_{2}$ and the following mapping, referred in [247] to as the gauge transformation

$$
x_{i} \mapsto y_{i}=\delta_{i} x_{i}, \quad \delta_{i}= \begin{cases}+1, & i \in V_{1} \\ -1, & i \in V_{2} .\end{cases}
$$

By noticing that $\delta_{i} \delta_{j} a_{i j}=\left|a_{i j}\right|$, (54) shapes into

$$
\dot{y}_{i}(t)=\sum_{j=1}^{n}\left|a_{i j}\right|\left(y_{j}(t)-y_{i}(t)\right) \quad \forall i,
$$

that is, the vector $y(t)$ obeys the Abelson model with the (nonnegative) weight matrix $A^{|\cdot|}=\left(\left|a_{i j}\right|\right)$ and

$$
L[A]=\Delta L\left[A^{|\cdot|}\right] \Delta, \quad \Delta=\Delta^{-1}=\operatorname{diag}\left(\delta_{1}, \ldots, \delta_{n}\right) .
$$

In particular, if the graph $\mathcal{G}$ has a directed spanning tree [1], then consensus is established in the system (56) $y_{i} \underset{t \rightarrow \infty}{\longrightarrow} p^{\top} y(0)$, where $p \geq 0$ is the only left eigenvector of $L\left[A^{|\cdot|}\right]$ such that $p^{\top} L\left[A^{|\cdot|}\right]=0$ and $p^{\top} \mathbb{1}_{n}=1$ (the vector of "social powers" [1]). This implies the following polarization property of the solutions, referred also to as the bipartite consensus [247]

$$
\lim _{t \rightarrow \infty} x_{i}(t)= \begin{cases}w^{\top} x(0), & i \in V_{1} \\ -w^{\top} x(0), & i \in V_{2},\end{cases}
$$

where $w=\Delta p$ is some non-zero vector (not necessarily positive). In other words, for almost all initial conditions opinions in the "hostile camps" reach consensus at the opposite values.

If $\mathcal{G}$ is structurally balanced but has no directed spanning tree, the opinions in (56) (and thus also in (54)) split into several clusters, determined by the graph's matrix of "spanning forests" [271].

Consider now the case of structurally imbalanced graph. In the case where $\mathcal{G}$ is strongly connected, Lemma 26 implies that the system (54) is exponentially stable since $(-L[A])$ is Hurwitz. In this case, opinions reach consensus at zero value independent 


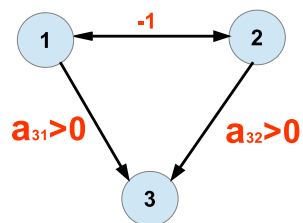

Figure 7: A structurally imbalanced quasi-strongly (yet not strongly) connected graph

of the initial condition. This statement is not valid for graphs with multiple strongly component component, as illustrated by the simple example with $n=3$ agents 264]. Let $a_{12}=a_{21}=-1$ and $a_{31}, a_{32}>0$, so the graph $\mathcal{G}$ has a directed spanning tree and is structurally imbalanced (Fig. 7). The Altafini system

$$
\begin{gathered}
\dot{x}_{1}=\left(-x_{2}-x_{1}\right), \dot{x}_{2}=\left(-x_{1}-x_{2}\right), \\
\dot{x}_{3}=a_{31} x_{1}+a_{32} x_{2}-\left(a_{31}+a_{32}\right) x_{3},
\end{gathered}
$$

has equilibria $(\xi,-\xi, \rho \xi)$, with $\rho=\left(a_{31}-a_{32}\right) /\left(a_{31}+\right.$ $\left.a_{32}\right) \in(-1 ; 1), \xi \in \mathbb{R}$, exhibiting thus neither polarization nor stability. Such a behavior is a special case of "interval bipartite consensu221, [272]. We do not consider the structure of the clusters, arising in (54) in the general case of imbalanced yet not strongly connected graphs and conditions, ensuring stability in this case, for further reading see [264, 272].

The results concerning the behavior of static Altafini's model are summarized in the following.

Theorem 29. For the Altafini model over a static graph $\mathcal{G}=(V, E, A)$, the following statements hold:

(a) if the graph has an directed spanning tree and is structurally balanced, the opinions polarize (57);

(b) if the graph is strongly connected and is structurally imbalanced, the system is stable;

(c) in general, the opinions converge and can split into several clusters, whose number and structure depend on the graph $\mathcal{G}$.

Remark 30. In the cases (a) and (b) opinions reach consensus in modulus in the sense that

$$
\lim _{t \rightarrow \infty}\left|x_{1}(t)\right|=\ldots=\lim _{t \rightarrow \infty}\left|x_{n}(t)\right| .
$$

\footnotetext{
${ }^{21}$ The work [272] deals with a quasi-strongly connected graphs with multiple root nodes (like nodes 1 and 2 in Fig. 7), constituting a closed strongly connected component [1] and reaching consensus in modulus (see Remark 30 below). Denoting their steady modulus value by $\theta=\theta(x(0)) \geq 0$, rhe "interval bipartite consensus" is defined in 272] as the convergence of all opinions $x_{i}(t)$ as $t \rightarrow \infty$ to the interval $[-\theta, \theta]$.
}

\subsubsection{The dynamic graph case}

The case of a general time-varying matrix $A(t)$ has not been fully studied, which is not surprising since necessary and sufficient conditions for convergence and consensus are still elusive even for the timevarying Abelson model $\left(a_{i j} \geq 0\right)$. Obviously, if the graph $\mathcal{G}[A(t)]$ is structurally balanced and the decomposition $V=V_{1} \cup V_{2}$ is time-invariant (that is, the friendship and enmity relations between each two agents are constant), the model reduces to Abelson model (56) via the gauge transformation (55). In general, such a transformation is not possible, nevertheless the properties of the Altafini model (54) and the associated Abelson model (56) are closely related.

The following counterpart of Lemma 5 has been established in [110, 264, 270].

Lemma 31. Suppose that the gains $a_{i j}(t)$ satisfy the following type-symmetry condition

$$
K^{-1}\left|a_{j i}(t)\right| \leq\left|a_{i j}(t)\right| \leq K\left|a_{j i}(t)\right| \quad \forall t \geq 0,
$$

where $K \geq 1$ is a constant. Then the limit $\xi_{i}=$ $\lim _{k \rightarrow \infty}\left|x_{i}(k)\right|$ exists for each $i$. If agents $i$ and $j$ interact persistently in the sense that

$$
\int_{0}^{\infty}\left|a_{i j}(t)\right| d t=\infty
$$

then the moduli of their final opinions coincide $\bar{\xi}_{i}=$ $\bar{\xi}_{j}$. If the graph of persistent interactions is connected, consensus in modulus (58) is established.

Similar to Lemma 5, Lemma 31 can be extended to graphs satisfying a more general cut-balance condition [110]. Lemma 31 implies, in particular, that if (59) holds consensus in (56) is equivalent to consensus in modulus in (54). This consensus in modulus can be either polarization (57) (with some $w \neq 0$ ) or asymptotic stability; necessity and sufficient conditions for both types of behavior has been given in [264, 270]. It is remarkable that Lemma 6] cannot be extended to Altafini's model in the same way: the UQSC condition for the matrix $A^{|\cdot|}$ implies consensus in (56), however, in general it implies neither consensus of the opinions' absolute values (58), nor even their convergence [264]. Consensus in modulus is provided by the uniform strong connectivity.

Lemma 32. Let $A(\cdot)$ be bounded $\left|a_{i j}(t)\right| \leq M$ and there exist $\varepsilon, T>0$ such that the following graphs

$$
\mathcal{G}_{\varepsilon, T}(t)=\mathcal{G}\left[\int_{t}^{t+T} A^{|\cdot|}(s) d s\right], \quad t \geq 0
$$


are strongly connected for any $t \geq 0$. Then consensus in modulus (58) is established.

Unlike the type-symmetric case from Lemma 31, in the situation of Lemma 32 it is not easy to give conditions for polarization and stability. To the best of the authors' knowledge, this problem has been solved only under additional assumptions [270, 273, 274].

\subsection{Extensions and related works}

In [275], a generalization of the Altafini models has been studied as follows

$$
\dot{x}_{i}(t)=-\sigma_{i} x_{i}(t)+\sum_{j=1}^{n} a_{i j} x_{j}(t),
$$

where $\sigma_{i}>0$ are constant "degradation rates", $a_{i i}=$ 0 and $a_{i j}$ can be both positive and negative for $i \neq j$. The main concern is the reaching of sign consensus, or "unanimity" [275] of opinions, that is, their convergence to the cone obtained by union of the positive and the negative orthant $\mathbb{R}_{+}^{n} \cup \mathbb{R}_{-}^{n}$. The key property, providing such a relaxed consensus, is eventual nonnegativity of the matrix $A$ (a matrix is eventually nonnegative if all its powers $A^{k}$ are non-zero and at least one of them is nonnegative). In [276], an interesting model has been proposed that combines the ideas of antagonistic interactions and bounded confidence (a smooth counterpart of this model has been also studied in [277] ). Extending the idea of gauge transformation, distributed continuous-time protocols sorting a given list of real numbers have been proposed in [278].

Along with continuous-time model (54), the "discrete-time Altafini model" can be considered

$$
x(k+1)=W(k) x(k) \in \mathbb{R}^{n},
$$

where the matrix $W^{|\cdot|}=\left(\left|w_{i j}(k)\right|\right)$ is stochastic and $w_{i i}(k) \geq 0$, while $w_{i j}(k)$ for $i \neq j$ can be negative. Properties of the discrete-time model (61) are similar to the properties of its continuous-time counterpart; e.g. consensus in modulus can be proved under the assumption of repeated strong connectivity. For this reason we do not consider the relevant theory and refer the reader to recent works [94, 273, 279, 280].

Among other extensions, gossip-based models with antagonistic interactions [281 283] should be mentioned. Unlike Altafini's model, some of these models [282, 283] provide polarization under the assumption of weak structural balance [266].

\section{Conclusions and Future Works}

The models describing social processes are numerous. It will not be an exaggeration to say that almost every week a novel model appears. When this tutorial was started, many of the papers referred in it had not been even written. Confining ourselves to a special class of dynamic models, we clearly realize that even this class remain partially uncovered by this tutorial. For instance, we do not consider models with quantized communication among the agents (that is, information an agent displays to the others is limited to a finite set of symbols) 284 286]. Focusing on stability and convergence problems, we avoid other important properties of opinion formation models such as e.g. their controllability [83, 287] and identifiability [288, 289]. Processes closely related to opinion formation, e.g. the dynamics of reflected appraisals [290 293], have been also excluded from the consideration. A very recent direction of research, opened by [294], deals with open models of multi-agent systems, which can be joined and left by agents. This approach opens up the perspective of modeling real social media, where agents interact via web forums, chats and blogs, and other temporal social networks that can loose and acquire not only connections among the nodes, but also nodes themselves. To make social networks more resilient against malicious attacks, it is important to understand mechanisms of "misbehavior" such as e.g. rumors, fake news and misinformation spreading [295, 296]. Models proposed to describe belief systems [297] and portraying the evolution of opinions on multiple interrelated topics [44, 203, 298, 299] also remain uncovered.

To overview all cutting-edge models in a journal paper is hardly possible, and without any doubts, sooner or later textbooks and monographs on social dynamics modeling will be published. The goal of this tutorial is to unveil the new field, lying in the frontier between systems theory and social science, to the broadest audience, and we hope that it will be helpful for researchers, starting working in this area.

In spite of recent progress in mathematical studies of opinion formation models, we still know very little about real processes in society. All of the existing models describe only one of the numerous facets of the social life, and most of focus on one special effect or property of social interactions (stubborness, homophily, xenophobia etc.) The ancient fable about blind men touching an elephant teaches us that this 
reductionist approach sometimes fails to give a real picture of the real phenomenon. Should we continue studying simplified models or seek for a complex holistic models of social influence? Which of the existing "simple" models is closer to reality? Should we use different models in different situations?

Evidently, mathematics cannot answer these questions; the only way to get realistic models is to work with data. Such data can be collected during social experiments. Nowadays, a huge amount of data is available in online social media. Although we witness some examples of successful interdisciplinary collaborations in experimental validation of dynamic models [206, 300], the current level of collaboration among between the communities systems and control theorists, sociologists and data scientists is insufficient. From our viewpoint, the toughest challenge is to unite the forces of these communities, elaborating a common language and instruments to be used in qualitative and quantitative studies of social dynamics.

\section{Afterword by Anton V. Proskurnikov}

This paper was conceived by Dr. Roberto Tempo and myself in 2016 as a survey, giving an overview of social dynamics models, scattered in mathematical, physical, sociological and engineering literature, from the systems and control viewpoint. Soon we realized that such a survey will be appreciated only by researchers, working on consensus and coordination of multi-agent networks, whereas our purpose was to open the exciting field of "social systems theory" to the broadest audience. It was decided to transform the survey into a tutorial, starting from the very beginning and introducing all necessary mathematical concepts. We have followed this plan in Part I, dealing with classical models of opinion formation.

Roberto's tragic and abrupt decease in January 2017 has made me postpone the submission of Part II for more than half a year and seriously restructure the text. Some sections, promised in Part I (e.g. distributed algorithms for network analysis and detailed discussion of emerging directions) have been discarded or shortened. Besides this, I have decided to include some very recent results, trying to keep a reasonable balance between tutorial and survey functions of this paper. Since the relevant mathematical techniques are too complicated to be considered, only some of the results are accompanied by full proofs.
Whether we like this or not, this tutorial is written by control theorists and primarily deals with problems, addressed by the systems and control community. I have received several emails, asking about the relation between the "strange" problems we consider and "actual" theory of social networks. Although I have done my best to explain the difference between the problems, models and approaches considered in this tutorial, and those studied by other communities (e.g. social network analysts, data scientists and statistical physicists), I have to repeat the key theses. We consider not social networks themselves, but rather dynamical processes over them, namely, dynamics of opinion formation. Among the dynamic models, we focus on agent-based models with continuous opinions, whereas statistical and discrete opinion models remain uncovered by this paper.

I am grateful to all colleagues, encouraging me to finish this important work, being one of the last Roberto's projects. I was especially encouraged by receiving messages from young researchers, urging me with impatience to publish its draft. I am indebted to Francoise Lamnabhi-Lagarrigue, Editor in Chief of Annual Reviews in Control, for inviting us to contribute this tutorial, and to my colleagues Noah Friedkin, Andreas Fläche, Francesco Bullo, Paolo Frasca, Claudio Altafini, Julien Hendrickx, Fabrizio Dabbene and Chiara Ravazzi for fruitful discussions.

\section{References}

\section{References}

[1] A. Proskurnikov, R. Tempo, A tutorial on modeling and analysis of dynamic social networks. Part I, Annual Reviews in Control 43, 65-79.

[2] J. Moreno, Who Shall Survive? A New Approach to the Problem of Human Interrelations, Nervous and Mental Disease Publishing Co., Washington, D.C., 1934.

[3] J. Moreno, Sociometry, Experimental Method, and the Science of Society, Beacon House, Ambler, PA, 1951.

[4] S. Wasserman, K. Faust, Social Network Analysis: Methods and Applications, Cambridge Univ. Press, Cambridge, 1994.

[5] J. Scott, Social Network Analysis, SAGE Publications, 2000.

[6] L. Freeman, The Development of Social Network Analysis. A Study in the Sociology of Science, Empirical Press, Vancouver, BC Canada, 2004.

[7] J. Scott, P. Carrington (Eds.), The SAGE Handbook on Social Network Analysis, SAGE Publications, 2011.

[8] D. Knoke, Political Networks. The Structural Perspective, Cambridge Univ. Press, 1993.

[9] D. Lazer, Networks in political science: Back to the future, PS: Political Science \& Politics 44 (1) (2011) 61-68. 
[10] A. O'Malley, P. Marsden, The analysis of social networks, Health Serv. Outcomes Res. Method. 8 (4) (2008) 222269.

[11] D. Easley, J. Kleinberg, Networks, Crowds and Markets. Reasoning about a Highly Connected World, Cambridge Univ. Press, Cambridge, 2010.

[12] M. Jackson (Ed.), Social and Economic Networks, Princeton Univ. Press, Princeton and Oxford, 2008.

[13] A. Masys (Ed.), Networks and Network Analysis for Defence and Security, Lecture Notes in Social Networks, Springer Int. Publ. Switzerland, 2014.

[14] G. Bichler, A. Malm (Eds.), Disrupting Criminal Networks: Network Analysis in Crime Prevention, Lynne Rienner Publ. Inc., 2015.

[15] V. Arnaboldi, A. Passarella, M. Conti, R. Dunbar, Online Social Networks. Human Cognitive Constraints in Facebook and Twitter Personal Graphs, Elsevier, New York, NY, 2015.

[16] P. Kazienko, N. Chawla (Eds.), Applications of Social Media and Social Network Analysis, Lecture Notes in Social Networks, Springer Int. Publ. Switzerland, 2015.

[17] S. Strogatz, Exploring complex networks, Nature 410 (2001) 268-276.

[18] M. Newman, The structure and function of complex networks, SIAM Review 45 (2) (2003) 167-256.

[19] M. Newman, A. Barabasi, D. Watts, The Structure and Dynamics of Networks, Princeton Univ. Press, 2006.

[20] P. Van Mieghem, Performance analysis of communications, networks and systems, Cambridge Univ. Press, 2006.

[21] R. Murray (Ed.), Control in an Information Rich World: Report of the Panel on Future Directions in Control, Dynamics and Systems, SIAM, Philadelphia, PA, 2003.

[22] T. Samad, A. Annaswamy (Eds.), The Impact of Control Technology. Overview, Success Stories, and Research Challenges, IEEE Control Systems Society, 2011.

[23] A. Annaswamy, T. Chai, S. Engell, S. Hara, A. Isaksson, P. Khargonekar, F. Lamnabhi-Lagarrigue, R. Murray, H. Nijmeijer, T. Samad, D. Tilbury, P. V. den Hof, Systems \& control for the future of humanity. Research agenda: current and future roles, impact and grand challenges, Annual Reviews in Control 43 (2017) 1-64.

[24] K. Lewin, Frontiers in group dynamics: Concept, method and reality in social science; social equilibria and social change, Human Relations 1 (5) (1947) 5-41.

[25] P. Sorokin, Society, Culture, and Personality: Their Structure and Dynamics, a System of General Sociology, Harper \& Brothers Publ., New York \& London, 1947.

[26] M. Diani, D. McAdam (Eds.), Social Movements and Networks, Oxford Univ. Press, Oxford, 2003.

[27] N. Wiener, The Human Use of Human Beings: Cybernetics and Society, Houghton Mifflin, New Haven, 1954.

[28] F. Geyer, The challenge of sociocybernetics, Kybernetes 24 (4) (1995) 6-32.

[29] F. Geyer, J. van der Zouwen (Eds.), Sociocybernetics: Complexity, autopoiesis, and observation of social systems, Greenwood Publ. Group, Westport, CT, USA, 2001.

[30] K. Bailey, Sociocybernetics and social entropy theory, Kybernetes 35 (3/4) (2006) 375-384.

[31] N. Friedkin, The problem of social control and coordination of complex systems in sociology: A look at the community cleavage problem, IEEE Control Syst. Mag. 35 (3) (2015) 40-51.

[32] J. Hołyst, K. Kacperski, F. Schweitzer, Social impact models of opinion dynamics, Annual Rev. Comput. Phys. 9 (06) (2001) 253-273.

[33] C. Castellano, S. Fortunato, V. Loreto, Statistical physics of social dynamics, Rev. Mod. Phys. 81 (2009) 591-646.

[34] D. Acemoglu, M. Dahleh, I. Lobel, A. Ozdaglar, Bayesian learning in social networks, Rev. Econom. Studies 78 (4) (2011) 1201-1236.

[35] H. Xia, H. Wang, Z. Xuan, Opinion dynamics: A multidisciplinary review and perspective on future research, Int. J. Knowledge Systems Sci. 2 (4) (2011) 72-91.

[36] Y. Dong, M. Zhan, G. Kou, Z. Ding, H. Liang, A survey on the fusion process in opinion dynamics, Information Fusion 43 (2018) 57-65.

[37] W. Ren, R. Beard, Distributed Consensus in MultiVehicle Cooperative Control: Theory and Applications, Springer-Verlag, London, 2008.

[38] W. Ren, Y. Cao, Distributed Coordination of Multiagent Networks, Springer, 2011.

[39] D. Knuth, Big Omicron and big Omega and big Theta, SIGACT News Apr.-Jun. (1976) 18-24.

[40] R. Abelson, Mathematical models of the distribution of attitudes under controversy, in: N. Frederiksen, H. Gulliksen (Eds.), Contributions to Mathematical Psychology, Holt, Rinehart \& Winston, Inc, New York, 1964, pp. $142-160$.

[41] J. Hunter, J. Danes, S. Cohen, Mathematical Models of Attitude Change, vol.1, Acad. Press, Inc., 1984.

[42] S. Kaplowitz, E. Fink, Dynamics of attitude change, in: R. Levine, H. Fitzgerald (Eds.), Analysis of Dynamic Psychological Systems. Vol. 2: Methods and Applications, Plenum Press, New York and London, 1992.

[43] J. Y. Halpern, The relationship between knowledge, belief, and certainty, Annals of Mathematics and Artificial Intelligence 4 (3) (1991) 301-322.

[44] N. Friedkin, A. Proskurnikov, R. Tempo, S. Parsegov, Network science on belief system dynamics under logic constraints, Science 354 (6310) (2016) 321-326.

[45] M. DeGroot, Reaching a consensus, Journal of the American Statistical Association 69 (1974) 118-121.

[46] L. Li, A. Scaglione, A. Swami, Q. Zhao, Consensus, polarization and clustering of opinions in social networks, IEEE J. on Selected Areas in Communications 31 (6) (2013) 1072-1083.

[47] P. Clifford, A. Sudbury, A model for spatial conflict, Biometrika 60 (3) (1973) 581-588.

[48] R. Holley, T. Liggett, Ergodic theorems for weakly interacting infinite systems and the voter model, The Annals of Probability 3 (4) (1975) 643-663.

[49] M. Granovetter, Threshold models of collective behavior, Amer. J. Sociology 83 (6) (1978) 1420-1443.

[50] J. Cox, D. Griffeath, Occupation time limit theorems for the voter model, Ann. Probab. 11 (4) (1983) 876-893.

[51] R. Axelrod, The dissemination of culture: A model with local convergence and global polarization, J. Conflict Resolut. 41 (1997) 203-226.

[52] K. Sznajd-Weron, J. Sznajd, Opinion evolution in closed community, Int. J. Mod. Phys. C 11 (06) (2000) 11571165.

[53] E. Yildiz, A. Ozdaglar, D. Acemoglu, A. Saberi, 
A. Scaglione, Binary opinion dynamics with stubborn agents, ACM Transactions on Economics and Computation 1 (4) (2013) article No. 19.

[54] Y. Dong, X. Chen, H. Liang, C.-C. Li, Dynamics of linguistic opinion formation in bounded confidence model, Information Fusion 32 (2016) 52-61.

[55] L. Tupikina, Dynamics on networks. Case of heterogeneous opinion status model, arXiv preprint 1708.01647.

[56] N. Rashevsky, Outline of a mathematical theory of human relations, Philosophy of Science 2 (4) (1935) 413430.

[57] N. Rashevsky, Studies in mathematical theory of human relations, Psychometrika 4 (3) (1939) 221-239.

[58] N. Rashevsky, Mathematical biophysics, Univ. Chicago Press, Chicago, IL, 1938.

[59] N. Rashevsky, Mathematical Theory of Human Relations, Principia Press, Bloomington, 1947.

[60] J. Jacquez, Compartmental analysis in biology and medicine, Univ. of Michigan Press, 1985.

[61] F. Brauer, P. van den Driessche, J. Wu (Eds.), Mathematical Epidemiology, Springer-Verlag, 2008.

[62] J. Maynard Smith, Evolution and the Theory of Games, Cambridge Univ. Press, 1982.

[63] W. Weidlich, The statistical description of polarization phenomena in society, Br. J. Math. Stat. Psychol. 24 (1971) 251-266.

[64] W. Weidlich, Thirty years of sociodynamics. An integrated strategy of modelling in the social sciences: applications to migration and urban evolution, Chaos, Solitons and Fractals 24 (2005) 45-56.

[65] D. Helbing, Quantitative Sociodynamics. Stochastic Methods and Models of Social Interaction Processes, Springer-Verlag Berlin Heidelberg, 2010.

[66] G. Como, F. Fagnani, Scaling limits for continuous opinion dynamics systems, The Annals of Applied Probability 21 (4) (2011) 5214-5240.

[67] C. Canuto, F. Fagnani, P. Tilli, An Eulerian approach to the analysis of Krause's consensus models, SIAM J. Control Optim. 50 (2012) 243-265.

[68] A. Mirtabatabaei, P. Jia, F. Bullo, Eulerian opinion dynamics with bounded confidence and exogenous inputs, SIAM J. Applied Dynam. Syst. 13 (1) (2014) 425-446.

[69] V. Blondel, J. Hendrickx, J. Tsitsiklis, On Krause's multiagent consensus model with state-dependent connectivity, IEEE Trans. Autom. Control 54 (11) (2009) 25862597.

[70] E. Wedin, P. Hegarty, The Hegselmann-Krause dynamics for the continuous-agent model and a regular opinion function do not always lead to consensus, IEEE Trans. Autom. Control 60 (9) (2015) 2416-2421.

[71] J. Hendrickx, A. Olshevsky, On symmetric continuum opinion dynamics, SIAM J. Control Optim. 54 (5) (2016) 2893-2918.

[72] R. Abelson, Mathematical models in social psychology, in: L. Berkowitz (Ed.), Advances in Experimental Social Psychology, v. 3, Acad. Press, New York, 1967, pp. 1-49.

[73] C. Reynolds, Flocks, herds, and schools: a distributed behavioral model, Computer Graphics 21 (4) (1987) 2534 .

[74] T. Vicsek, A. Czirok, E. Ben-Jacob, O. Cohen, I. Shochet, Novel type of phase transition in a system of self-driven particles, Phys. Rev. Letters 75 (96) (1995)
1226-1229.

[75] S. Strogatz, Sync: The Emerging Science of Spontaneous Order, Hyperion Press, New York, 2003.

[76] J. French Jr., A formal theory of social power, Physchol. Rev. 63 (1956) 181-194.

[77] F. Harary, A criterion for unanimity in French's theory of social power, in: D. Cartwright (Ed.), Studies in Social Power, Univ. of Michigan Press, Ann Arbor, MI, 1959, pp. $168-182$.

[78] F. Harary, R. Norman, D. Cartwright, Structural Models. An Introduction to the Theory of Directed Graphs, Wiley \& Sons, New York, London, Sydney, 1965.

[79] F. Bullo, Lectures on Network Systems, published online at http://motion.me.ucsb.edu/book-lns, 2016, with contributions by J. Cortes, F. Dorfler, and S. Martinez.

[80] T. Kurahashi-Nakamura, M. Mäs, J. Lorenz, Robust clustering in generalized bounded confidence models, J. Artificial Societies and Social Simulation 19 (4) (2016) 7.

[81] D. Watts, Six Degrees: The Science of a Connected Age, Norton, New York, 2003.

[82] R. Hegselmann, U. Krause, Opinion dynamics under the influence of radical groups, charismatic leaders and other constant signals: a simple unifying model, Networks and Heterogeneous Media 10 (3) (2015) 477-509.

[83] N. Masuda, Opinion control in complex networks, New J. Phys. 17 (3) (2015) 033031.

[84] N. Friedkin, E. Johnsen, Social influence networks and opinion change, in: S. Thye, E. Lawler, M. Macy, H. Walker (Eds.), Advances in Group Processes, Vol. 16, 1999, pp. 1-29.

[85] N. Friedkin, E. Johnsen, Social Influence Network Theory, Cambridge Univ. Press, New York, 2011.

[86] R. Olfati-Saber, J. Fax, R. Murray, Consensus and cooperation in networked multi-agent systems, Proceedings of the IEEE 95 (1) (2007) 215-233.

[87] Z. Li, Z. Duan, G. Chen, L. Huang, Consensus of multiagent systems and synchronization of complex networks: A unified viewpoint, IEEE Trans. on Circuits and Systems - I 57 (1) (2010) 213-224.

[88] C. Wu, Synchronization in complex networks of nonlinear dynamical systems, World Scientific, Singapore, 2007.

[89] A. Proskurnikov, M. Cao, Consensus in multi-agent systems, in: J. Webster (Ed.), Wiley Encyclopedia of Electrical and Electronics Engineering, Wiley \& Sons, 2016.

[90] A. Proskurnikov, A. Fradkov, Problems and methods of network control, Autom. Remote Control 77 (10) (2016) 1711-1740.

[91] V. Blondel, J. Hendrickx, A. Olshevsky, J. Tsitsiklis, Convergence in multiagent coordination, consensus, and flocking, in: Proc. IEEE Conf. Decision and Control, 2005, pp. $2996-3000$.

[92] L. Moreau, Stability of multiagent systems with timedependent communication links, IEEE Trans. Autom. Control 50 (2) (2005) 169-182.

[93] J. Lorenz, A stabilization theorem for dynamics of continuous opinions, Physica A 353 (2005) 217-223.

[94] A. Proskurnikov, M. Cao, Modulus consensus in discretetime signed networks and properties of special recurrent inequalities, in: Proc. of IEEE Conf. Decision and Control, 2017, pp. 2003-2008.

[95] G. Shi, K. Johansson, The role of persistent graphs in the agreement seeking of social networks, IEEE J. On 
Selected Areas In Communications 31 (9) (2013) 595606.

[96] W. Xia, G. Shi, Z. Meng, M. Cao, K. Johansson, Discrete-time consensus systems under persistent flow and non-instantaneous reciprocal interactions. Online at https://arxiv.org/abs/1705.07532.

[97] A. Proskurnikov, R. Tempo, M. Cao, N. Friedkin, Opinion evolution in time-varying social influence networks with prejudiced agents, IFAC PapersOnline 50 (1) (2017) 11896-11901.

[98] B. Chazelle, The total s-energy of a multiagent system, SIAM J. Control Optim. 49 (4) (2011) 1680-1706.

[99] J. Hajnal, Weak ergodicity in non-homogeneous Markov chains, Proc. Cambridge Philos. Soc. 54 (1958) 233-246.

[100] J. Wolfowitz, Products of indecomposable, aperiodic, stochastic matrices, Proceedings of Amer. Math. Soc. 15 (1963) 733-737.

[101] S. Chatterjee, E. Seneta, Towards consensus: Some convergence theorems on repeated averaging, Journal of Applied Probability 14 (1977) 89-97.

[102] J. Lorenz, Consensus strikes back in the HegselmannKrause model of continuous opinion dynamics under bounded confidence, Journal of Artifical Societies and Social Simulation (JASSS) 9 (1) (2006) 8.

[103] J. Hendrickx, J. Tsitsiklis, Convergence of typesymmetric and cut-balanced consensus seeking systems, IEEE Trans. Autom. Control 58 (1) (2013) 214-218.

[104] M. Cao, A. Morse, B. Anderson, Reaching a consensus in a dynamically changing environment: a graphical approach, SIAM J. Control Optim. 47 (2) (2008) 575-600.

[105] D. Angeli, P. Bliman, Stability of leaderless discrete-time multi-agent systems, Math. Control, Signals, Syst. 18 (4) (2006) 293-322.

[106] A. Proskurnikov, Average consensus in networks with nonlinearly delayed couplings and switching topology, Automatica 49 (9) (2013) 2928-2932.

[107] A. Matveev, I. Novinitsyn, A. Proskurnikov, Stability of continuous-time consensus algorithms for switching networks with bidirectional interaction, in: Proc. Europ. Control Conference (ECC), 2013, pp. 1872-1877.

[108] G. Shi, K. Johansson, Robust consensus for continuoustime multi-agent dynamics, SIAM J. Control Optim 51 (5) (2013) 3673-3691.

[109] S. Martin, J. Hendrickx, Continuous-time consensus under non-instantaneous reciprocity, IEEE Trans. Autom. Control 61 (9) (2016) 2484-2495.

[110] A. Proskurnikov, M. Cao, Differential inequalities in multi-agent coordination and opinion dynamics modeling, Automatica 85 (2017) 202-210.

[111] L. Moreau, Stability of continuous-time distributed consensus algorithms, in: Proc. IEEE Conf. Decision and Control (CDC 2004), 2004, pp. 3998 - 4003.

[112] Z. Lin, B. Francis, M. Maggiore, State agreement for continuous-time coupled nonlinear systems, SIAM J. Control Optim. 46 (1) (2007) 288-307.

[113] U. Münz, A. Papachristodoulou, F. Allgöwer, Consensus in multi-agent systems with coupling delays and switching topology, IEEE Trans. Autom. Control 56 (12) (2011) 2976-2982.

[114] A. Proskurnikov, Nonlinear consensus algorithms with uncertain couplings, Asian Journal of Control 16 (5) (2014) 1277-1288.
[115] A. Proskurnikov, A. Matveev, Popov-type criterion for consensus in nonlinearly coupled networks, IEEE Trans. Cybernetics 45 (8) (2015) 1537-1548.

[116] A. Proskurnikov, F. Zhang, M. Cao, J. Scherpen, A general criterion for synchronization of incrementally dissipative nonlinearly coupled agents, in: Proceedings of European Control Conference (ECC 2015), Linz, Austria, 2015, pp. 581-586.

[117] A. Papachristodoulou, A. Jadbabaie, U.Münz, Effects of delay in multi-agent consensus and oscillator synchronization, IEEE Trans. Autom. Control 55 (6) (2010) 1471 $-1477$.

[118] M. McPherson, L. Smith-Lovin, J. Cook, Birds of a feather: Homophily in social networks, Annu. Rev. Sociol. 27 (2001) 415444.

[119] N. Mark, Culture and competition: Homophily and distancing explanations for cultural niches, Amer. Soc. Review 68 (3) (2003) 319-345.

[120] P. Dandekar, A. Goel, D. Lee, Biased assimilation, homophily, and the dynamics of polarization, PNAS 110 (15) (2013) 5791-5796.

[121] M. Mäs, A. Flache, D. Helbing, Individualization as driving force of clustering phenomena in humans, PLoS Comput. Biol. 6 (10) (2010) e1000959.

[122] U. Krause, A discrete nonlinear and non-autonomous model of consensus formation, Commun. in Difference Equations (2000) 227-236.

[123] G. Deffuant, D. Neau, F. Amblard, G. Weisbuch, Mixing beliefs among interacting agents, Advances in Complex Systems 3 (2001) 87-98.

[124] R. Hegselmann, U. Krause, Opinion dynamics and bounded confidence models, analysis, and simulation, Journal of Artifical Societies and Social Simulation (JASSS) 5 (3) (2002) 2.

[125] J. Herrera, M. Cosenza, K. Tucci, J. González-Avella, General coevolution of topology and dynamics in networks, EPL (Europhys. Letters) 95 (5) (2011) 58006.

[126] F. Cucker, S. Smale, Emergent behavior in flocks, IEEE Trans. Automat. Control 52 (5) (2007) 852-862.

[127] R. Olfati-Saber, Flocking for multi-agent dynamic systems: algorithms and theory, IEEE Trans. Autom. Control 51 (3) (2006) 401-420.

[128] H. Tanner, A. Jadbabaie, G. Pappas, Flocking in fixed and switching networks, IEEE Trans. Autom. Control 52 (5) (2007) 863-868.

[129] F. Bullo, J. Cortes, S. Martinez, Distributed control of robotics networks, Princeton Univ. Press, 2009.

[130] V. Blondel, J. Hendrickx, J. Tsitsiklis, On the 2R conjecture for multi-agent systems, in: Proc. of European Control Conf. (ECC), 2007, pp. 874-881.

[131] C. Wang, Q. Li, E. Weinan, B. Chazelle, Noisy Hegselmann-Krause systems: Phase transition and the 2R-conjecture, J. Stat. Phys. 166 (2017) 1209-1225.

[132] J. Dittmer, Consensus formation under bounded confidence, Nonlinear Analysis 47 (7) (2001) 4615-4621.

[133] A. Bhattacharyya, M. Braverman, B. Chazelle, H. Nguyen, On the convergence of the HegselmannKrause system, in: Proc. 4th Conf. on Innovations in Theoretical Computer Science, 2013, pp. 61-66.

[134] S. Mohajer, B. Touri, On convergence rate of scalar Hegselmann-Krause dynamics, in: Proc. Amer. Control Conf. (ACC), 2013, pp. 206-210. 
[135] S. Etesami, T. Başar, Game-theoretic analysis of the Hegselmann-Krause model for opinion dynamics in finite dimensions, IEEE Trans. Autom. Control 60 (7) (2015) 1886-1897.

[136] B. Touri, A. Nedić, Discrete-time opinion dynamics, in: 45th Conf. on Signals, Systems and Computers (ASILOMAR), 2011, pp. 1172-1176.

[137] E. Wedin, P. Hegarty, A quadratic lower bound for the convergence rate in the one-dimensional HegselmannKrause bounded confidence dynamics, Discrete Comput. Geom. 53 (2015) 478-486.

[138] A. Nedić, B. Touri, Multi-dimensional HegselmannKrause dynamics, in: Proc. of IEEE Conf. on Decision and Control (CDC), 2012, pp. 68-73.

[139] S. Etesami, T. Başar, A. Nedić, B. Touri, Termination time of multidimensional Hegselmann-Krause opinion dynamics, in: Proc. of the American Control Conference (ACC), 2013, pp. 1255-1260.

[140] A. Martinsson, An improved energy argument for the Hegselmann-Krause model, J. of Difference Equations and Applications 22 (4) (2016) 630-635.

[141] B. Touri, Product of random stochastic matrices and distributed averaging (Ph.D. Thesis), Univ. of Illinois at Urbana-Champaign, Urbana, IL, 2011.

[142] P.-E. Jabin, S. Motsch, Clustering and asymptotic behavior in opinion formation, J. Differential Equations 257 (11) (2014) 4165-4187.

[143] S. Motsch, E. Tadmor, Heterophilious dynamics enhances consensus, SIAM Review 56 (4) (2013) 577-621.

[144] S. Chen, D. Glass, M. McCartney, Characteristics of successful opinion leaders in a bounded confidence model, Physica A 449 (2016) 426-436.

[145] M. Roozbehani, A. Megretski, E. Frazzoli, Lyapunov analysis of quadratically symmetric neighborhood consensus algorithms, in: Proc. of IEEE Conf. Decision and Control (CDC), 2008, pp. 2252-2257.

[146] J. Coulson, D. Steeves, B. Gharesifard, B. Touri, On the termination time of the one-sided asymmetric Hegselmann-Krause dynamics, in: Proc. of American Control Conference (ACC), 2015, pp. 4054-4059.

[147] V. Blondel, J. Hendrickx, J. Tsitsiklis, Continuoustime average-preserving opinion dynamics with opiniondependent communications, SIAM J. Control Optim. 48 (2010) 5214-5240.

[148] J. Lorenz, Continuous opinion dynamics under bounded confidence: a survey, Int. J. of Modern Phys. C 18 (12) (2007) 1819-1838.

[149] S. Fortunato, V. Latora, A. Pluchino, A. Rapisarda, Vector opinion dynamics in a bounded confidence consensus model, Int. J. of Modern Phys. C 16 (2005) 1535-1551.

[150] Y. Yang, D. Dimarogonas, X. Hu, Opinion consensus of modified Hegselmann-Krause models, Automatica 50 (2) (2014) 622-627.

[151] F. Ceragioli, P. Frasca, Continuous and discontinuous opinion dynamics with bounded confidence, Nonlinear Analysis: Real World Applications 13 (3) (2012) 12391251.

[152] S. Stamoulas, M. Rathinam, Convergence, stability and robustness of multidimensional opinion dynamics in continuous time, online at http://arxiv.org/abs/1507.05530.

[153] R. Hegselmann, U. Krause, Truth and cognitive division of labour: First steps towards a computer aided social epistemology, Journal of Artifical Societies and Social Simulation (JASSS) 9 (3) (2006) 1.

[154] S. Kurz, J. Rambau, On the Hegselmann-Krause conjecture in opinion dynamics, J. Difference Equations and Applications 17 (6) (2011) 859-876.

[155] B. Chazelle, C. Wang, Inertial Hegselmann-Krause systems, IEEE Trans. Autom. Control 62 (8) (2017) 39053913.

[156] D. Urbig, J. Lorenz, H. Herzberg, Opinion dynamics: The effect of the number of peers met at once, J. Artificial Societies and Social Simulation (JASSS) 11 (2) (2008) 1.

[157] G. Fu, W. Zhang, Z. Li, Opinion dynamics of modified Hegselmann-Krause model in a group-based population with heterogeneous bounded confidence, Physica A 419 (2015) 558-565.

[158] A. Mirtabatabaei, F. Bullo, Opinion dynamics in heterogeneous networks: Convergence conjectures and theorems, SIAM J. Control Optim. 50 (5) (2012) 2763-2785.

[159] J. Lorenz, Heterogeneous bounds of confidence: Meet, discuss and find consensus, Complexity 15 (4) (2010) 4352.

[160] Y. Zhao, L. Zhang, M. Tang, G. Kou, Bounded confidence opinion dynamics with opinion leaders and environmental noises, Computers Operations Research 74 (2016) 205-213.

[161] D. Tangredi, R. Iervolino, F. Vasca, Consensus stability in the Hegselmann-Krause model with coopetition and cooperosity, IFAC-PapersOnLine 50 (1) (2017) 1192011925.

[162] R. Iervolino, D. Tangredi, F. Vasca, Piecewise quadratic stability of consensus in heterogeneous opinion dynamics, in: Proc. of European Control Conference (ECC), 2016, pp. 549-554.

[163] W. Su, G. Chen, Y. Hong, Noise leads to quasi-consensus of Hegselmann-Krause opinion dynamics, Automatica 85 (2017) 448-454.

[164] M. Pineda, R. Toral, E. Hernández-García, The noisy Hegselmann-Krause model for opinion dynamics, Eur. Phys. J. B 86 (2013) 490.

[165] F. Baccelli, A. Chatterjee, S. Vishwanath, Stochastic bounded confidence opinion dynamics, in: Proc. of the IEEE Conf. on Decision and Control (CDC), 2014, pp. 3408-3413.

[166] J. Zhang, Y. Hong, X. Hu, Multiagent opinion dynamics of bounded confidence with nonlocal aggregative interaction, SIAM J. Control Optim. 55 (4) (2017) 2543-2573.

[167] I. Douven, A. Riegler, Extending the HegselmannKrause model I, Logic Journal of the IGPL 18 (2) (2010) 323-335.

[168] I. Douven, A. Riegler, Extending the HegselmannKrause model II, in: T. Czarnecki, K. Kijania-Placek, O. Poller, J. Wolenski (Eds.), The Analytical Way, College Publ., London, 2010, pp. 245-258.

[169] I. C. Morarescu, A. Girard, Opinion dynamics with decaying confidence: Application to community detection in graphs, IEEE Transactions on Automatic Control 56 (8) (2011) 1862-1873.

[170] K. Varshney, Bounded confidence opinion dynamics in a social network of bayesian decision makers, IEEE J. Selected Topics Signal Process. 8 (4) (2014) 576-585.

[171] G. Oliva, D. LaManna, A. Fagiolini, R. Setola, Dis- 
tributed data clustering via opinion dynamics, Int. J. Distributed Sensor Networks (2015) 753102.

[172] H. Liang, Y. Dong, C.-C. Li, Dynamics of uncertain opinion formation: An agent-based simulation, J. Artificial Societies and Social Simulation (JASSS) 19 (4) (2016) 1.

[173] I. Douven, A. Riegler, Extending the HegselmannKrause model III: From single beliefs to complex belief states, Episteme 6 (2) (2009) 145-163.

[174] B. Chazelle, Natural algorithms and influence systems, Commun. of the ACM 55 (12) (2012) 101-110.

[175] B. Chazelle, Diffusive influence systems, SIAM J. Comput. 44 (5) (2015) 1403-1442.

[176] B. Chazelle, An algorithmic approach to collective behavior, J. Stat. Phys. 158 (2015) 514-548.

[177] F. Fagnani, S. Zampieri, Randomized consensus algorithms over large scale networks, IEEE J. Selected Areas Commun. 26 (4) (2008) 634-649.

[178] R. Tijdeman, On a telephone problem, Nieuw Archief voor Wiskunde 19 (3) (1971) 188-192.

[179] A. Hajnal, E. Milner, E. Szemerédi, A cure for the telephone disease, Canad. Math. Bull. 15 (3) (1972) 447-450.

[180] B. Baker, R. Shostak, Gossips and telephones, Discrete Math. 2 (3) (1972) 191-193.

[181] F. Harary, A. Schwenk, The communication problem on graphs and digraphs, J. Franklin Inst. 297 (1974) 491495.

[182] F. Harary, A. Schwenk, Efficiency of dissemination of information in one-way and two-way communication networks, Syst. Res. Behavioral Sci. 19 (2) (1974) 133-135.

[183] S. Hedetniemi, S. Hedetniemi, A. Liestman, A survey of gossiping and broadcasting in communication networks, Networks 18 (4) (1988) 319-349.

[184] J. Liu, S. Mou, A. Morse, B. Anderson, C. Yu, Deterministic gossiping, Proc. IEEE 99 (9) (2011) 1505-1524.

[185] M. Cao, A. Morse, B. Anderson, Reaching a consensus in a dynamically changing environment: convergence rates, measurement delays, and asynchronous events, SIAM J. Control Optim. 47 (2) (2008) 601-623.

[186] M. Cao, A. Morse, B. Anderson, Agreeing asynchronously, IEEE Trans. Autom. Control 53 (8) (2008) 1826-1838.

[187] A. Shiriaev, Probability, Springer, 1996.

[188] A. Tahbaz-Salehi, A. Jadbabaie, A necessary and sufficient condition for consensus over random networks, IEEE Trans. Autom. Control 53 (3) (2008) 791-795.

[189] Q. Song, G. Chen, D. Ho, On the equivalence and condition of different consensus over a random network generated by i.i.d. stochastic matrices, IEEE Trans. Autom. Control 56 (5) (2011) 1203-1207.

[190] Y. Hatano, M. Mesbahi, Agreement over random networks, IEEE Trans. Autom. Control 50 (11) (2005) 18671872 .

[191] C. Wu, Synchronization and convergence of linear dynamics in random directed networks, IEEE Trans. Autom. Control 51 (7) (2006) 1207-1210.

[192] A. Tahbaz-Salehi, A. Jadbabaie, Consensus over ergodic stationary graph processess, IEEE Trans. Autom. Control 55 (1) (2010) 225-230.

[193] B. Touri, A. Nedić, On ergodicity, infinite flow, and consensus in random models, IEEE Trans. Autom. Control 56 (7) (2011) 1593-1605.

[194] S. Boyd, A. Ghosh, B. Prabhakar, D. Shah, Randomized gossip algorithms, IEEE Trans. Inform. Theory 52 (6) (2006) 2508-2530.

[195] T. Aysal, M. Yildiz, A. Sarwate, A. Scaglione, Broadcast gossip algorithms for consensus, IEEE Trans. Signal Proc. 57 (7) (2009) 2748-2761.

[196] A. Dimakis, S. Kar, J. Moura, M. Rabbat, A. Scaglione, Gossip algorithms for distributed signal processing, Proc. of the IEEE 98 (11) (2010) 1847-1863.

[197] J. Liu, B. Anderson, M. Cao, A. Morse, Analysis of accelerated gossip algorithms, Automatica 49 (4) (2013) 873-883.

[198] D. Acemoglu, G. Como, F. Fagnani, A. Ozdaglar, Opinion fluctuations and disagreement in social networks, Math. of Oper. Research 38 (1) (2013) 1-27.

[199] P. Frasca, C. Ravazzi, R. Tempo, H. Ishii, Gossips and prejudices: Ergodic randomized dynamics in social networks, in: Proc. of IFAC NecSys 2013 Workshop, Koblenz, Germany, 2013, pp. 212-219.

[200] C. Ravazzi, P. Frasca, R. Tempo, H. Ishii, Ergodic randomized algorithms and dynamics over networks, IEEE Trans. Control of Network Syst. 2 (1) (2015) 78-87.

[201] P. Frasca, H. Ishii, C. Ravazzi, R. Tempo, Distributed randomized algorithms for opinion formation, centrality computation and power systems estimation: A tutorial overview, Europ. J. Control 24 (7) (2015) 2-13.

[202] S. Parsegov, A. Proskurnikov, R. Tempo, N. Friedkin, A new model of opinion dynamics for social actors with multiple interdependent attitudes and prejudices, in: Proc. of IEEE Conference on Decision and Control (CDC), 2015, pp. $3475-3480$.

[203] S. Parsegov, A. Proskurnikov, R. Tempo, N. Friedkin, Novel multidimensional models of opinion dynamics in social networks, IEEE Trans. Autom. Control 62 (5) (2017) 2270-2285.

[204] Y. Peres, W. Schlag, B. Solomyak, Sixty years of Bernoulli convolutions, in: C. Bandt, S. Graf, M. Zähle (Eds.), Fractal Geometry and Stochastics II, Birkhäuser, Basel, 2000.

[205] C. Childress, N. Friedkin, Cultural reception and production, Amer. Soc. Rev. 77 (2012) 45-68.

[206] N. Friedkin, F. Bullo, How truth wins in opinion dynamics along issue sequences, PNAS 114 (43) (2017) 1138011385 .

[207] H. Ishii, R. Tempo, Distributed randomized algorithms for the PageRank computation, IEEE Trans. Autom. Control 55 (9) (2010) 1987-2002.

[208] H. Ishii, R. Tempo, The PageRank problem, multi-agent consensus and Web aggregation: A systems and control viewpoint, IEEE Control Syst. Mag. 34 (3) (2014) 34-53.

[209] K. You, R. Tempo, L. Qiu, Distributed algorithms for computation of centrality measures in complex networks, IEEE Trans. Autom. Control 62 (5) (2017) 2080-2094.

[210] G. Deffuant, D. Neau, F. Amblard, G. Weisbuch, How can extremism prevail? A study based on the relative agreement interaction model, Journal of Artifical Societies and Social Simulation (JASSS) 5 (4) (2002) 1.

[211] G. Deffuant, Comparing extremism propagation patterns in continuous opinion models, Journal of Artifical Societies and Social Simulation (JASSS) 9 (3) (2006) 8.

[212] G. Weisbuch, Bounded confidence and social networks, Eur. Phys. J. B 38 (2004) 339-343.

[213] G. Weisbuch, G. Deffuant, F. Amblard, Persuasion dy- 
namics, Physica A 353 (2005) 555-575.

[214] E. Kurmyshev, H. Juárez, R. Gonzalez-Silva, Dynamics of bounded confidence opinion in heterogeneous social networks: Concord against partial antagonism, Physica A 390 (2011) 2945-2955.

[215] N. Abrica-Jacinto, E. Kurmyshev, H. Juárez, Effects of the interaction between ideological affinity and psychological reaction of agents on the opinion dynamics in a relative agreement model, J. Artificial Societies and Social Simulation (JASSS) 20 (3) (2017) 3.

[216] J. Zhang, Y. Hong, Opinion evolution analysis for shortrange and long-range DeffuantWeisbuch models, Physica A 392 (2013) 5289-5297.

[217] J. Zhang, G. Chen, Convergence rate of the asymmetric Deffuant-Weisbuch dynamics, J Syst Sci Complex 28 (2015) 558-565.

[218] J.-D. Mathias, S. Huet, G. Deffuant, Bounded confidence model with fixed uncertainties and extremists: The opinions can keep fluctuating indefinitely, J. Artificial Societies and Social Simulation (JASSS) 19 (1) (2016) 6.

[219] F. Amblard, G. Deffuant, The role of network topology on extremism propagation with the relative agreement opinion dynamics, Physica A 343 (2004) 725-738.

[220] G. Weisbuch, G. Deffuant, F. Amblard, J.-P. Nadal, Meet, discuss, and segregate!, Complexity 7 (3) (2002) $55-63$.

[221] Y. Shang, An agent based model for opinion dynamics with random confidence threshold, Commun. Nonlin. Sci. Numer. Simulat. 19 (2014) 3766-3777.

[222] A. Carro, R. Toral, M. San Miguel, The role of noise and initial conditions in the asymptotic solution of a bounded confidence, continuous-opinion model, J. Stat. Phys. 151 (2013) 131-149.

[223] F. Baccelli, A. Chatterjee, S. Vishwanath, Pairwise stochastic bounded confidence opinion dynamics: Heavy tails and stability 62 (11) (2017) 5678-5693.

[224] K. Malarz, Sociocybernetics and social entropy theory, Int. J. Modern Phys. C 17 (10) (2006) 1521-1524.

[225] M. Pineda, G. Buendía, Mass media and heterogeneous bounds of confidence in continuous opinion dynamics, Physica A 420 (2015) 73-84.

[226] G. Pache, Coopetitive supply chains: Toward a social ties perspective, Management 18 (2) (2013) 125-140.

[227] J. Hu, W. Zheng, Emergent collective behaviors on coopetition networks, Phys. Lett. A 378 (2014) 17871796.

[228] K. Coyte, J. Schluter, K. Foster, The ecology of the microbiome: Networks, competition, and stability, Science 350 (6261) (2015) 663-666.

[229] C. Samaniego, G. Giordano, F. Blanchini, E. Franco, Stability analysis of an artificial biomolecular oscillator with non-cooperative regulatory interaction, J. Biol. Dynam. 11 (1) (2017) 102-120.

[230] P. Romanczuk, L. Schimansky-Geier, Swarming and pattern formation due to selective attraction and repulsion, Interface Focus 2 (2012) 746-756.

[231] W. Yu, G. Chen, M. Cao, J. Lü, H. Zhang, Swarming behaviors in multi-agent systems with nonlinear dynamics, Chaos 23 (2013) 043118.

[232] A. Zakhar'eva, A. S. Matveev, M. C. Hoy, A. V. Savkin, Distributed control of multiple non-holonomic robots with sector vision and range-only measurements for target capturing with collision avoidance, Robotica 33 (2015) 385-412.

[233] C. Hovland, I. Janis, H. Kelley, Communication and Persuasion, Yale Univ. Press, New Haven, 1953.

[234] A. Allahverdyan, A. Galstyan, Opinion dynamics with confirmation bias, PLoS One 9 (7) (2014) e99557.

[235] R. Abelson, J. Miller, Negative persuasion via personal insult, J. Experim. Soc. Psychol. 3 (1967) 321-333.

[236] J. Dillard, L. Shen, On the nature of reactance and its role in persuasive health communication, Communication Monographs 72 (2) (2005) 144-168.

[237] F. Heider, Attitudes and cognitive organization, J. Psychol. 21 (1946) 107-122.

[238] L. Festinger, A Theory of Cognitive Dissonance, Stanford Univ. Press, Stanford, CA, 1957.

[239] K. Takács, A. Fläche, M. Mäs, Discrepancy and disliking do not induce negative opinion shifts, PLoS One 11 (6) (2016) e0157948.

[240] A. Flache, M. Mäs, How to get the timing right? a computational model of how demographic faultlines undermine team performance and how the right timing of contacts can solve the problem, Comput. Math. Organ. Theory 14 (2008) 23-51.

[241] A. Fläche, M. Macy, Small worlds and cultural polarization, Journal of Math. Sociology 35 (1-3) (2011) 146-176.

[242] T. Feliciani, A. Flache, J. Tolsma, How, when and where can spatial segregation induce opinion polarization? two competing models, J. Artificial Societies and Social Simulation (JASSS) 20 (2) (2017) 6.

[243] M. W. Macy, J. Kitts, A. Flache, S. Benard, Polarization in dynamic networks: a Hopfield model of emergent structure, in: R. Breiger, K. Carley, P. Pattison (Eds.), Dynamic Social Network Modeling and Analysis: Workshop Summary and Papers, 2003, pp. 162-173.

[244] D. Baldassarri, P. Bearman, Dynamics of political polarization, Amer. Sociol. Rev. 72 (2007) 784-811.

[245] L. Salzarulo, A continuous opinion dynamics model based on the principle of meta-contrast, Journal of Artifical Societies and Social Simulation (JASSS) 9 (1) (2006) 13.

[246] C. Altafini, Dynamics of opinion forming in structurally balanced social networks, PLoS ONE 7 (6) (2012) e38135.

[247] C. Altafini, Consensus problems on networks with antagonistic interactions, IEEE Trans. Autom. Control 58 (4) (2013) 935-946.

[248] M. Valcher, P. Misra, On the consensus and bipartite consensus in high-order multi-agent dynamical systems with antagonistic interactions, Systems Control Letters 66 (2014) 94-103.

[249] M.-C. Fan, H.-T. Zhang, M. Wang, Bipartite flocking for multi-agent systems, Commun Nonlinear Sci Numer Simulat 19 (2014) 3313-3322.

[250] S. Zhai, Modulus synchronization in a network of nonlinear systems with antagonistic interactions and switching topologies, Commun. Nonlin. Sci. Numer. Simulat. 33 (2016) 184-193.

[251] F. Heider, Social perception and phenomenal causality, Psychol. Rev. 51 (1944) 358-374.

[252] D. Cartwright, F. Harary, Structural balance: A generalization of Heider's theory, Physchol. Rev. 63 (1956) 277-293.

[253] T. Schwartz, The friend of my enemy is my enemy, the enemy of my enemy is my friend: Axioms for structural 
balance and bi-polarity, Mathematical Social Sciences 60 (2010) 39-45.

[254] G. Facchetti, G. Iacono, C. Altafini, Computing global structural balance in large-scale signed social networks, PNAS 108 (52) (2011) 20953-20958.

[255] X. Zheng, D. Zeng, F.-Y. Wang, Social balance in signed networks, Inform. Syst. Frontiers 17 (5) (2015) 10771095.

[256] N. Hummon, P. Doreian, Some dynamics of social balance processes: bringing Heider back into balance theory, Social Networks 25 (2003) 17-49.

[257] T. Antal, P. Krapivsky, S. Redner, Dynamics of social balance on networks, Phys. Rev. E 72 (2005) 036121.

[258] T. Antal, P. Krapivsky, S. Redner, Social balance on networks: the dynamics of friendship and enmity, Physica D 224 (2006) 130-136.

[259] K. Kulakowski, Some recent attempts to simulate the Heider balance problem, Computing in Science and Engineering 9 (2007) 80-85.

[260] P. Abell, M. Ludwig, Structural balance: a dynamic perspective, J. Math. Soc. 33 (2009) 129-135.

[261] S. Marvel, J. Kleinberg, R. Kleinberg, S. Strogatz, Continuous-time model of structural balance, PNAS 108 (5) (2011) 1771-1776.

[262] P. Jia, N. Friedkin, F. Bullo, The coevolution of appraisal and influence networks leads to structural balance, IEEE Trans. Network Science Engineering 3 (4) (2016) 286298.

[263] A. Parravano, A. Andina-Díaz, M. Meléndez-Jiménez, Bounded confidence under preferential flip: A coupled dynamics of structural balance and opinions, PLoS One 11 (10) (2016) e0164323.

[264] A. Proskurnikov, A. Matveev, M. Cao, Opinion dynamics in social networks with hostile camps: Consensus vs. polarization, IEEE Trans. Autom. Control 61 (6) (2016) 1524-1536.

[265] F. Harary, On the notion of balance of a signed graph, Michigan Math. J. 2 (1953) 143-146.

[266] J. Davis, Clustering and structural balance in graphs, Human Relations 20 (1967) 181-187.

[267] T. Zaslavsky, Balanced decompositions of a signed graph, J. Combinatorial Theory. Series B. 43 (1987) 1-13.

[268] A. Proskurnikov, A. Matveev, M. Cao, Consensus and polarization in Altafini's model with bidirectional timevarying network topologies, in: Proceedings of IEEE CDC 2014, Los Angeles, CA, 2014, pp. 2112-2117.

[269] A. Proskurnikov, M. Cao, Opinion dynamics using Altafini's model with a time-varying directed graph, in: Proceedings of IEEE ISIC 2014 (Part of IEEE MSC 2014), Antibes, 2014, pp. 849-854.

[270] J. Hendrickx, A lifting approach to models of opinion dynamics with antagonisms, in: Proc. of IEEE Conference on Decision and Control (CDC), 2014, pp. 2118-2123.

[271] P. Chebotarev, R. Agaev, The forest consensus theorem, IEEE Trans. Autom. Control 59 (9) (2014) 2475-2479.

[272] D. Meng, J. Du, Y. Jia, Interval bipartite consensus of networked agents associated with signed digraphs, IEEE Trans. Autom. Control 61 (12) (2016) 3755-3770.

[273] J. Liu, X. Chen, T. Basar, M. Belabbas, Exponential convergence of the discrete- and continuous-time Altafini models, IEEE Trans. Autom. Control 62 (12) (2017) 6168-6182.
[274] D. Meng, Z. Meng, Y. Hong, Uniform convergence for signed networks under directed switching topologies, Automatica 90 (2018) 8-15.

[275] C. Altafini, G. Lini, Predictable dynamics of opinion forming for networks with antagonistic interactions, IEEE Trans. on Autom. Control 60 (2) (2015) 342-357.

[276] F. Ceragioli, G. Lindmark, C. Veibäck, N. Wahlström, M. Lindfors, C. Altafini, A bounded confidence model that preserves the signs of the opinions, in: Proc. of European Control Conference (ECC), 2016, pp. 543-548.

[277] A. Proskurnikov, M. Cao, A novel homogenous protocol for multi-agent clustering over directed graphs, in: Proc. American Control Conference (ACC), Boston, MA, 2016, pp. 1906-1911.

[278] C. Altafini, A continuous-time dynamical system that can sort agents through distributed protocols, in: Proc. of IEEE Conference on Control Applications (CCA), 2014, pp. 2153-2158.

[279] W. Xia, M. Cao, K. Johansson, Structural balance and opinion separation in trust-mistrust social networks, IEEE Trans. on Control of Networks 3 (1) (2016) 46-56.

[280] Z. Meng, G. Shi, K. Johansson, M. Cao, Y. Hong, Behaviors of networks with antagonistic interactions and switching topologies, Automatica 73 (2016) 110-116.

[281] G. Shi, M. Johansson, K. Johansson, How agreement and disagreement evolve over random dynamic networks, IEEE J. On Selected Areas In Communications 31 (6) (2013) 1061-1071.

[282] G. Shi, A. Proutiere, M. Johansson, J. Baras, K. Johansson, Emergent behaviors over signed random dynamical networks: State-flipping model, IEEE Trans. on Control of Networked Systems 2 (2) (2015) 142-153.

[283] G. Shi, A. Proutiere, M. Johansson, J. Baras, K. Johansson, The evolution of beliefs over signed social networks, Oper. Res. 64 (3) (2016) 585-604.

[284] D. Urbig, Attitude dynamics with limited verbalisation capabilities, Journal of Artifical Societies and Social Simulation (JASSS) 6 (1) (2003) 2.

[285] A. Martins, Trust in the CODA model: Opinion dynamics and the reliability of other agents, Physica A 377 (37) (2013) 2333-2339.

[286] F. Ceragioli, P. Frasca, Consensus and disagreement: The role of quantized behaviors in opinion dynamics, SIAM J. Control Optim. 56 (2) (2018) 1058-1080.

[287] F. Dietrich, S. Martin, M. Jungers, Control via leadership of opinion dynamics with state and time-dependent interactions, IEEE Trans. Autom. Control, published online under DOI 10.1109/TAC.2017.2742139.

[288] H.-T. Wai, A. Scaglione, A. Leshem, Active sensing of social networks, IEEE Trans. Signal Inform. Process. Netw. 2 (3) (2016) 406-419.

[289] C. Ravazzi, R. Tempo, F. Dabbene, Learning influence structure in sparse social networks, IEEE Trans. Control Netw. Syst., published online under DOI 10.1109/TCNS.2017.2781367.

[290] P. Jia, A. Mirtabatabaei, N. Friedkin, F. Bullo, Opinion dynamics and the evolution of social power in influence networks, SIAM Review 57 (3) (2015) 367-397.

[291] N. Friedkin, P. Jia, F. Bullo, A theory of the evolution of social power: natural trajectories of interpersonal influence systems along issue sequences, Soc. Science 3 (2016) $444-472$. 
[292] X. Chen, J. Liu, M.-A. Belabbas, Z. Xu, T. Başar, Distributed evaluation and convergence of self-appraisals in social networks, IEEE Trans. Autom. Control 62 (1) (2017) 291-304.

[293] M. Ye, J. Liu, B. Anderson, C. Yu, T. Başar, On the analysis of the DeGroot-Friedkin model with dynamic relative interaction matrices, IFAC-PapersOnLine 50 (1) (2017) 11902-11907.

[294] J. M. Hendrickx, S. Martin, Open multi-agent systems: Gossiping with random arrivals and departures, in: Proc. of IEEE Conf. Decision and Control, 2017, pp. 763-768.

[295] D. Acemoglu, A. Ozdaglar, Opinion dynamics and learning in social networks, Dyn. Games Appl. 1 (2011) 3-49.

[296] M. Del Vicario, A. Bessi, F. Zollo, F. Petroni, A. Scala, G. Caldarelli, H. E. Stanley, W. Quattrociocchi, The spreading of misinformation online, PNAS 113 (3) (2016) 554-559.

[297] P. Converse, The nature of belief systems in mass publics, in: D. Apter (Ed.), Ideology and Discontent, Free Press, New York, 1964, pp. 206-261.

[298] F. Xiong, Y. Liu, L. Wang, X. Wang, Analysis and application of opinion model with multiple topic interactions, Chaos 27 (2017) 083113.

[299] H. Noorazar, M. Sottile, K. Vixie, A energy-based interaction model for population opinion dynamics with topic coupling, arXiv preprint 1607.06806.

[300] C. Vande Kerckhove, S. Martin, P. Gend, P. Rentfrow, J. Hendrickx, V. Blondel, Modelling influence and opinion evolution in online collective behaviour, PLoS One 11 (6) (2016) e0157685. 